J3eA 5 (2006)

Doi: 10.1051/j3ea:2006021

\title{
e-Learning en génie électrique : Développement coopératif de ressources pédagogiques multimédia
}

\author{
Par Sophie Labrique ${ }^{1}$, Damien Grenier ${ }^{2}$, Sergiu Ivanov $^{3}$, Francis Labrique ${ }^{1}$, Maria-José \\ Resende ${ }^{4}$, Benoît Robyns ${ }^{5}$ \\ $1 \quad$ Université catholique de Louvain \\ 2 ENS de Cachan (Antenne de Bretagne) \\ $3 \quad$ Facultatea de Electromecanica, Universitatea din Craiova, Roumanie \\ $4 \quad$ Instituto Superior Técnico , Lisbonne, Portugal \\ $5 \quad$ Ecole des Hautes Etudes d’Ingénieur (HEI)
}

Résumé : Le génie électrique est une discipline difficile à apprendre et à enseigner. Elle exige en effet, de la part des étudiants, un niveau d'abstraction beaucoup plus poussé que dans d'autres disciplines. L'apparition des technologies de l'information et de la communication (TIC) offre l'opportunité de revoir les approches pédagogiques et les rendre plus efficaces. Dans ce but, un site internet d'e-learning en génie électrique est développé par quatre Universités et Grandes Ecoles de Belgique, de France, du Portugal et de Roumanie, avec le soutien de l'Union Européenne. Cet article présente ce projet, quelques réalisations du site, un certain nombre d'outils et méthodes informatiques partagés par les partenaires du projet et la création d'une association sans but lucratif ayant pour objectif de poursuivre et d'élargir l'expérience.

Mots clés : e-Learning, génie électrique, circuits électriques, électronique de puissance, énergies renouvelables, machines électriques

Titre court : e-Learning en génie électrique

\section{Les auteurs}

Sophie Labrique est diplômée Ingénieur Civil Architecte de l'Université catholique de Louvain (UCL), Belgique, en 1995. De 1996 à 1999, elle travaille comme architecte collaborateur auprès du bureau d'architecture ARCHI+I, situé à Bruxelles. En 1999, elle est engagée comme assistante de recherche à l'UCL pour réaliser un site internet pour l'enseignement des circuits électriques et des convertisseurs électromécaniques. Depuis 2003, elle supervise les développements informatiques dans le cadre du projet e-LEE (e-Learning tools for Electrical Engineering).

Damien Grenier, est un ancien élève de l'ENS de Cachan où il a obtenu son doctorat en 1994. Après un séjour post-doctoral au sein du Groupe de Recherche en Electronique de Puissance et Commande Industrielle (GREPCI) de l'Ecole de Technologie Supérieure (ETS), Montréal, Québec, Canada, il rejoint en 1996 l’Université catholique de Louvain où il 
développe entre autres des activités dans le domaine du e-learning. Il est coordonnateur du projet européen Socrates-Minerva «e-LEE ». Depuis septembre 2005, il est professeur des université à l'Antenne de Bretagne de l'ENS de Cachan.

Sergiu Ivanov, est diplômé "Ingénieur Civil Electricien" et "Docteur en Génie Electrique"de l'Université de Craiova (UCv), Roumanie, respectivement en 1986 et 1998. De 1986 à 1991 il a travaillé comme chercheur à l'Institut de Recherche pour les Moteurs, les Transformateurs et les Equipements Electriques, à Craiova. En 1991 il a rejoint la Faculté d’Electromécanique de l'Université de Craiova. Il est responsable des cours "Commande vectorielle des entraînements électriques » et "Modélisation et simulation des équipements électriques ». Il est également Secrétaire Scientifique de la Faculté d’Electromécanique de l’Université de Craiova.

Francis Labrique, est diplômé Ingénieur Civil Electricien et Docteur en Sciences Appliquées de l'Université catholique de Louvain. Il a effectué sa carrière au département d'Electricité de cette même université où il est actuellement professeur et responsable du Laboratoire d'Electrotechnique et d'Instrumentation. De 1985 à 1991, il a été Professeur Associé Invité au département de Génie Electrique et Ordinateurs de l'Instituto Superior Técnico de Lisbonne. Il est Docteur Honoris Causa de l'université de Craiova (UCv), Roumanie.

Maria-José Resende, est diplômée "Ingénieur Electricien" et "Docteur en Sciences Appliquées" de l' Instituto Superior Técnico (IST) de la Universidade Técnica de Lisboa (UTL), Portugal, respectivement en 1986 et 1998. Elle est engagée au Département Génie Electrique et Ordinateurs de l'Instituto Superior Técnico depuis 1986 où elle est, actuellement, Professeur Assistant. Elle est effectue sa recherche au sein du Centro de Automática da Universidade Técnica de Lisboa (CAUTL) depuis 1986.

Benoît Robyns est diplômé "Ingénieur Civil Electricien" et "Docteur en Sciences appliquées" de l'Université Catholique de Louvain (UCL), Belgique, respectivement en 1987 et 1993. En 2000, il reçoit le diplôme d' "Habilitation à Diriger des Recherches" de l'Université des Sciences et Technologies de Lille (USTL). Il est responsable du Département Génie Electrique de l'Ecole des Hautes Etudes d'Ingénieur (HEI) de Lille. Il est aussi responsable de l'équipe Réseaux Electriques et Systèmes Energétiques (RESE) du Laboratoire d'Electrotechnique et d'Electronique de Puissance (L2EP) de Lille.

\section{Introduction}

Le génie électrique est la branche de la physique appliquée qui traite de la mise en œuvre, dans des dispositifs fabriqués par l'homme, de phénomènes électriques et magnétiques. Cette discipline couvre des domaines d'applications qui se sont sans cesse élargis au cours du 20ème siècle et continuent à faire l'objet de développements importants dans des secteurs d'activités stratégiques, comme l'électronique, les systèmes de télécommunication et la gestion de l'énergie. Les besoins en terme de main d'œuvre qualifiée dans ce domaine sont énormes. Pourtant, on constate une désaffection constante des étudiants qui rechignent à choisir cette filière. 
Il est vrai que le génie électrique est une discipline difficile à apprendre (et à enseigner !). Elle exige en effet, de la part des étudiants, un niveau d'abstraction beaucoup plus élévé que dans d'autres disciplines. En mécanique les notions élémentaires de position, de vitesse, de forces, ... sont des notions intuitives, correspondant à des expériences vécues par tout un chacun et sur lesquelles l'enseignant peut s'appuyer pour introduire les concepts de base. Les lois qui régissent un dispositif mécanique sont faciles à visualiser, car elles peuvent être illustrées au moyen d'expériences qui montrent de manière directe les relations mises en jeu. En revanche, nul n'a jamais vu une charge, un courant ou un potentiel électrique autrement que par les effets qu'ils produisent. Ces grandeurs ne peuvent être visualisées qu'au travers d'une instrumentation (voltmètre, ampèremètre, oscilloscope) qui mesure les valeurs de certains paramètres et ne donnent qu'une vision indirecte des phénomènes physiques en jeu. Dans les machines électriques ces grandeurs évoluent de plus simultanément dans le temps et dans l'espace. Comprendre dès lors comment elles peuvent interagir pour créer des efforts mécaniques est loin d'être immédiat.

Les technologies de l'information et de la communication (TIC) s'avèrent alors des outils précieux. En permettant aux étudiants de visualiser l'évolution des grandeurs physiques au sein des dispositifs électriques, elles rendent plus intuitive la compréhension des phénomènes qui s’y déroulent et facilitent la compréhension des modèles mathématiques qui les décrivent rigoureusement. Par la possibilité qu'elles offrent de manipuler virtuellement ces systèmes, elles facilitent leur appropriation par les étudiants. Cela se fait en outre sans risque de dégradation d'équipements souvent coûteux, ni d'électrocution et permet aux étudiants d'expérimenter plus tôt (même si les bases élémentaires ne sont pas toutes acquises) et plus souvent dans leur formation.

Dans cet esprit, un site internet d'e-learning en génie électrique (www.e-lee.net) est développé par quatre Universités et Grandes Ecoles de Belgique, de France, du Portugal et de Roumanie, avec le soutien de l'Union Européenne (Figure 1). Cet article présente ce projet, brièvement quelques ressources développées sur le site, les méthodes et outils informatiques mis en œuvre pour leur réalisation et la création d'une association sans but lucratif ayant pour objectif de poursuivre et élargir l’expérience. 

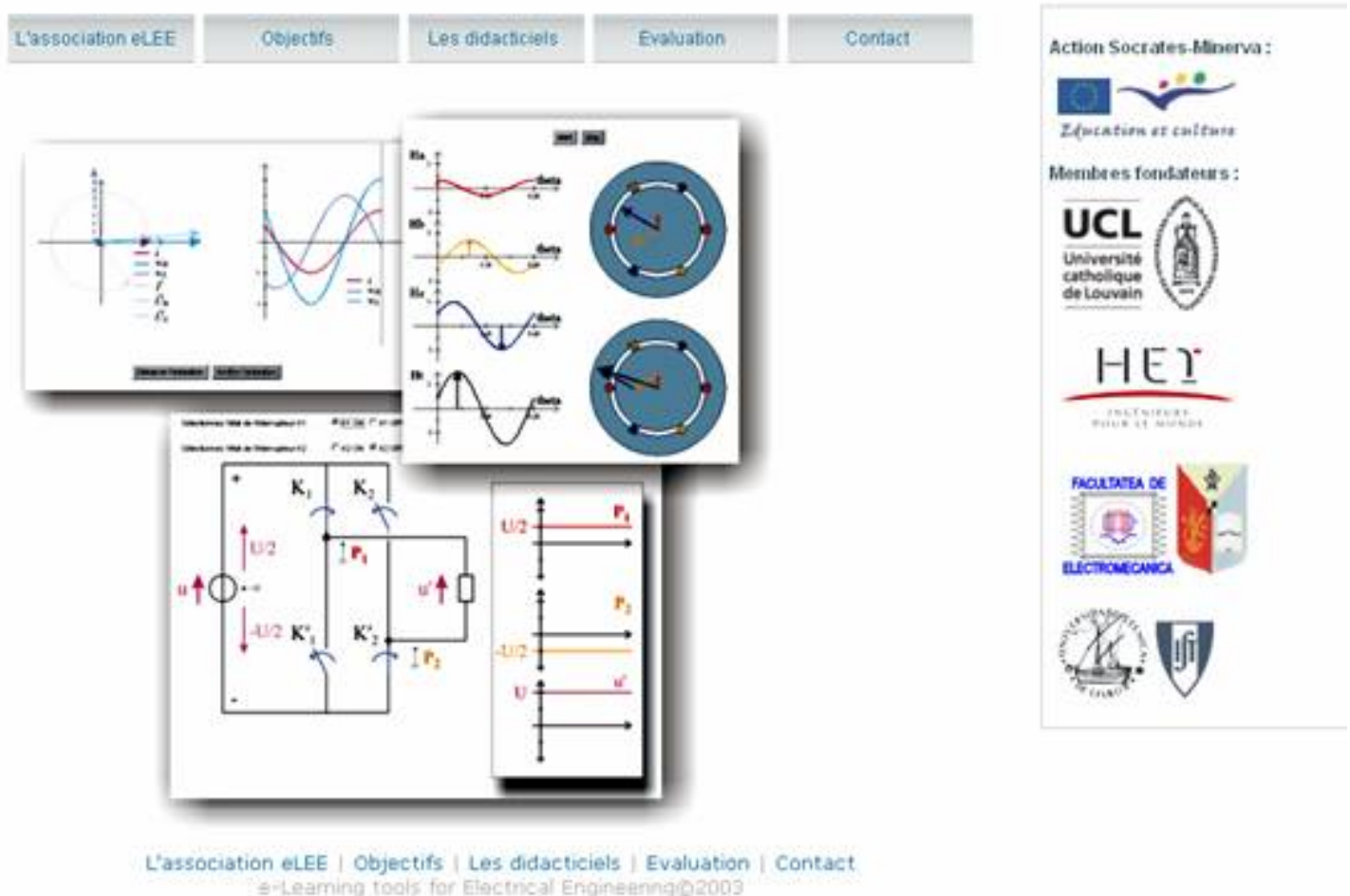

Figure 1. Page d'accueil du site "www.e-lee.net"

\section{Un projet européen}

Les outils pédagogiques nés des technologies de l'information et de la communication peuvent apporter une aide indéniable aux étudiants pour la compréhension des matières concernées. C'est en tout cas ce qui ressort d'une première expérience menée à l’Université catholique de Louvain (UCL), Belgique, où les étudiants invités à évaluer les outils réalisés en soutien d'un cours d'électrotechnique [2], avaient surtout mis en avant l'intérêt de l'interactivité proposée par de tels outils [3].

L'apparition des TIC offre en effet l'opportunité de revoir les approches pédagogiques conventionnelles et de les rendre plus efficaces. Il ne faut cependant pas se bercer d'illusions. Tout comme l'invention de l'imprimerie n'a pas fait disparaître dans les pratiques d'enseignement l'exposé oral et la prise de note qui lui est liée, tout comme le développement de l'audiovisuel ne s'est pas fait au détriment du support écrit, les TIC ne révolutionneront pas le métier d'enseignant. Elles ne s'implanteront que là où elles sont capables d'apporter un véritable plus aux étudiants, en les aidant, plus que tout autre moyen, à comprendre et s’approprier la matière à étudier.

Nous avons au fur et à mesure du développement des ressources et de leur publication sur le réseau, interrogé nos étudiants sur l'usage qu'ils faisaient des outils proposés, sur l'aide que cela leur apportait,... Plus de 400 étudiants ont répondu aux questionnaires d'évaluation qui 
leurs étaient soumis et une trentaine d'entre eux ont été interrogés individuellement par un expert externe à l'équipe de développement [4].

Les étudiants privilégient, parmi toutes les ressources disponibles, les contenus les plus interactifs (questionnaires d'auto-évaluation, exercices corrigés, laboratoires virtuels, animations, ...) qui leur apportent une plus-value par rapport aux moyens pédagogiques usuels (cours, laboratoires et exercices). Ils ne considèrent toutefois pas pour autant qu'ils peuvent entièrement se substituer à ces derniers : ils préfèrent la leçon en auditoire ou un texte à lire sur papier (éventuellement distribué par le web) aux leçons en lignes... si celles-ci ne comportent pas d'animations.

Partant de ce constat, les concepteurs du site www.e-lee.net ont focalisé leurs efforts sur le développement de ressources qui ne se substituent pas aux supports de cours traditionnels, mais offrent à l'étudiant l'occasion d'appréhender de façon plus active et interactive certains points de la matière.

La conception et le développement de telles ressources interactives entraînent un investissement extrêmement coûteux en terme de ressources humaines. Sous peine de voir les ressources développées devenir rapidement obsolètes, leur maintenance, leur adaptation à l'évolution continuelle et rapide des technologies informatiques mises en œuvre, représentent des coûts non moins élevés à long terme. Il s'agit en tout cas d'un investissement qui contrairement à celui de la rédaction d'un texte de cours (polycopié, livre) n'est pas à la porté d'un enseignant ou même d'un établissement isolé.

Le développement de coopérations en vue de la réalisation de ressources pédagogiques multimédia communes paraît dès lors la seule solution réaliste pour permettre l'intégration de ces nouveaux outils au sein de nos dispositifs pédagogiques. Plus le réseau de coopération sera développé, plus les coûts de réalisation et de maintenance pourront être facilement amortis. Se pose toutefois alors la question de savoir si une ressource développée par un autre établissement, dont le niveau des étudiants, les objectifs en terme de formation, les approches pédagogiques diffèrent, peut être utilisée telle quelle et si non, quelles adaptations sont nécessaires.

C'est en partie pour répondre à cette question que le projet e-LEE (e-learning tools for Electrical Engineering) a été initié et subsidié par la commission européenne dans le cadre de l'action Socrates-Minerva. Quatre universités et grandes écoles : l’Université catholique de Louvain (UCL, Belgique), qui assure la coordination du projet, l'Ecole des Hautes Études d’Ingénieur (HEI, Lille, France), l’Instituto Superior Técnico (IST, Lisbonne, Portugal) et la Facultatea de Electromecanica de l'Université de Craiova (UCv, Roumanie) tentent l'expérience d'un développement coopératif de ressources multimédia dans 4 thématiques: circuits électriques, électronique de puissance, machines électriques et énergies renouvelables. Les ressources sont déclinées en 4 langues : français, anglais, portugais et roumain. Elles sont accessibles gratuitement à tout internaute au travers du site www.e-lee.net.

Cet article présente quelques réalisations du site, le partage entre les partenaires du projet d'une "e-learning toolbox" qui regroupe des méthodes et outils informatiques d'aide à la conception de ressources pédagogiques et, suite aux premiers résultat de l'évaluation de l'impact de tels outils auprès des étudiants, la création d'une association ayant pour objectif de poursuivre et d'élargir l'expérience. 


\section{Un site internet}

\section{Types de ressources}

Différents types de ressources pédagogiques sont proposées sur le site.

1. les « leçons » en ligne, davantage dans l'esprit d'un exposé au tableau que d'un cours rédigé sur un support papier, illustrent certaines notions théoriques au moyen d'animations, de photos et/ou de courtes séquences vidéo ;

2. les «laboratoires virtuels " proposent des exercices résolus, des expériences virtuelles, ou des simulations. Chaque laboratoire contient l'énoncé du problème et une série de questions. L'étudiant est invité à chercher lui-même la solution par un cheminement progressif. Ainsi, dans certains cas, un bouton d'aide rappelle un théorème ou une formule utiles, suggère des hypothèses simplificatrices. La réponse à la question est d'abord donnée sous forme d'un résultat brut, afin que l'étudiant qui n'a pas obtenu la bonne réponse puisse reprendre son raisonnement ou ses calculs. L'illustration de cette réponse au moyen d'un graphe animé ou d'un résultat de simulation aide à identifier d'éventuelles erreurs de raisonnement. Vient finalement la démonstration complète de la réponse, éventuellement par différentes méthodes (la méthode la plus élégante, car concise, présentée dans les ouvrages de référence n'est pas forcément la seule méthode acceptable). Enfin, une vérification de la réponse est parfois proposée, sous forme d'un résultat expérimental ou d'une simulation par un modèle moins simplificateur que celui considéré dans le calcul ;

3. les modules d'auto-évaluation, sous forme de questionnaires à choix multiple (QCM), par le biais d'une correction automatique, permettent à l'apprenant de vérifier et de mesurer son niveau de connaissance et de compréhension de la matière traitée dans le chapitre.

\section{Interactivité proposée}

Ces ressources regroupent plusieurs types d'outils pédagogiques qui, tous, proposent à l'étudiant une plus ou moins grande interactivité.

L'étudiant navigue à travers les pages web et aborde la matière à son propre rythme et éventuellement selon un cheminement qui lui est propre. La présentation progressive de chaque point de la matière, la résolution "étape par étape " des exercices, la possibilité d'obtenir une aide ponctuelle ou de consulter le moteur de recherche du site, les liens hypertextes vers d'autres points abordés précédemment et nécessaires à une bonne compréhension, sont autant de jalons qui aident l'étudiant à compléter et structurer ses connaissances, à mettre en évidence et combler les « zones d'ombres » qui subsistent dans son apprentissage de la matière.

L'étudiant teste et évalue son niveau de connaissance: il s'essaie à la résolution de questionnaires à choix multiple, un module de correction automatique repère ses erreurs et le renvoie vers un texte d'explication. Le score obtenu lui donne une indication de sa connaissance de la matière.

Enfin et surtout, grâce aux animations, l'étudiant manipule virtuellement les dispositifs qu'il est en train d'étudier (ou du moins leur simulation). L'interactivité est dans ce cas maximale : 
- alors qu'il est usuel dans un ouvrage sur support papier ou au tableau lors d'un cours, de tracer les courbes qui caractérisent le fonctionnement d'un dispositif pour un jeu de paramètres bien choisi, le support électronique permet à l'étudiant de voir comment celles-ci sont affectées (ou non) par une modification des paramètres internes ou externes du dispositif.

- I'animation des figures est d'ailleurs un outil irremplaçable pour visualiser la manière dont les grandeurs caractéristiques des dispositifs étudiés évoluent simultanément dans le temps et dans l'espace.

- les outils de simulation de dispositifs électriques contribuent à la réalisation de laboratoires virtuels. Ces laboratoires, s'ils ne prétendent pas remplacer la confrontation indispensable avec la réalité, multiplient les occasions offertes aux étudiants de manipuler les dispositifs étudiés et ainsi de se les approprier. II leur est même possible d'apprendre en faisant des erreurs, en "cassant" virtuellement des machines, choses que pour des raisons de coût et de sécurité il est impossible de leur laisser faire dans un laboratoire réel. Enfin, cela leur permet de manipuler des dispositifs dans des gammes de puissance qu'aucune installation didactique ne pourra jamais atteindre.

\section{Les réalisations}

Grâce au financement européen, des ressources sont actuellement développées autour de 4 thématiques. Chaque partenaire a pris en charge la responsabilité du développement d'une thématique.

- circuits électriques (IST, Lisbonne, Portugal) : description et illustration des méthodes de base pour l'écriture et la résolution des équations des circuits électriques (de la loi des mailles et loi des nœuds jusqu'aux méthodes vectorielles pour la résolution des circuits alternatifs en passant par les théorèmes de Thevenin-Norton et de superposition) ;

- électronique de puissance (UCL, Louvain-la-Neuve, Belgique) : description et illustration du fonctionnement des principales structures permettant la conversion d'énergie électrique à l'aide de semi-conducteurs de puissance fonctionnant en commutation ;

- machines asynchrones (UCV, Craiova, Roumanie): construction, mise en équation, fonctionnement à fréquence fixe (alimentation à partir du réseau) ou à fréquence variable (commande U/f, vectorielle, DTC) ;

- énergies renouvelables (HEI, Lille, France) : description technique et illustration des filières de production d'énergie électrique à partir de sources renouvelables, dont les filières éoliennes et photovoltaïques.

Tout ou partie de ces ressources sont développées, traduites et adaptées en 4 langues : roumain, portugais, français et anglais.

Les deux premiers secteurs correspondent à des disciplines de base de l'électricité. Ils permettent de considérer deux des principales méthodes d'analyse des circuits électriques. Les deux derniers secteurs correspondent à des domaines beaucoup plus pointus et pluridisciplinaires. L'un très technique concerne des étudiants très spécialisés dans le domaine. Les énergies renouvelables sont en revanche un secteur d'application qui peut intéresser un public beaucoup plus large. 
Nous présentons ci-après quelques une des réalisations les plus emblématiques parmi celles développées au sein de ces 4 thématiques.

Circuits électriques : représentation vectorielle des grandeurs sinusoïdales.

Les ingénieurs électrotechniciens ont l'habitude d'analyse le régime permanent des circuits et dispositifs alimentés par des sources sinusoïdales, en utilisant une représentation vectorielle des courants et tensions (phaseurs ou diagrammes de Fresnel...). Quand le professeur tente d'expliquer au cours combien il est plus simple pour analyser ces circuits d'associer à chaque tension ou courant une grandeur complexe (dont la partie réelle représente la véritable de la tension ou du courant) et de représenter graphiquement chacune de ces grandeurs par un vecteur tournant dans un espace à deux dimensions, il ressent au mieux une certaine inquiétude et de l'incrédulité chez les étudiants ! Le professeur peut tenter d'argumenter à coup d'équations et de schémas au tableau mais il n'aura jamais la force de conviction d'une animation multimédia que l'étudiant peut utiliser pour reconstruire par luimême tous les schémas que le professeur a dessiné au tableau, en y introduisant en plus la composante temps (la rotation des vecteurs à la fréquence de la source et l'évolution sinusoïdale de leur projection sur un axe), ce qu'aucun schéma imprimé ou tracé au tableau ne peut

faire.

La «leçon en ligne» sur les grandeurs sinusoïdales commence par la définition des paramètres qui caractérisent ce type de grandeurs : amplitude, pulsation et phase. Une animation permet à l'étudiant de faire varier chacun de ces paramètres en visualisant leur influence sur le signal produit, à l'aide d'un oscilloscope virtuel.

Figure 2a. Visualisation des paramètres caractérisant une grandeur sinusoïdale (Animation disponible sur le site http://dx.doi.org/10.1051/j3ea:2006021)

Vient ensuite la notion de valeur efficace, avec sa traduction physique et graphique.

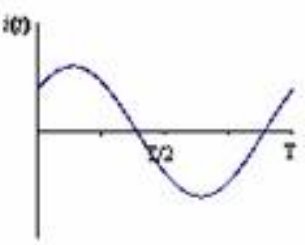

$i(t)$

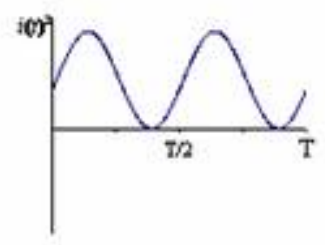

$[i(t)]^{]}$
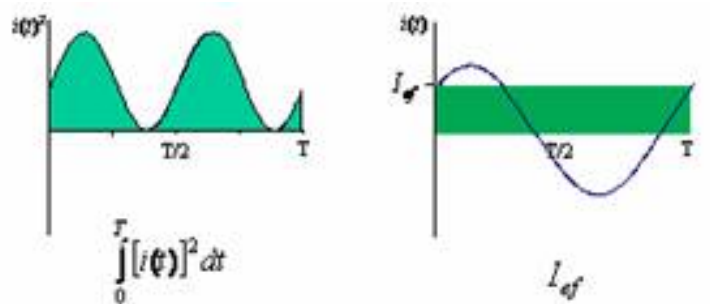

Figure 2b. Visualisation de la notion de valeur efficace

On montre enfin comment une grandeur caractérisée par l'expression : $x(t)=X_{M} \sin (\omega t+\varphi)$

peut être représentée symboliquement par un vecteur (une grandeur complexe) $\bar{X}_{M}=X_{M} e^{j(a t+\varphi)}$

qui tourne dans le plan avec une pulsation ${ }^{\omega}$. La projection, à chaque instant, de ce vecteur tournant sur l'axe réel correspond à la valeur instantanée, à cet instant, de la grandeur sinusoïdale. L'animation montre à la fois le vecteur tournant et sa projection sur l'axe, en laissant à l'étudiant la possibilité de modifier les paramètres caractérisant la grandeur. 
Figure 2c. Représentation d'une grandeur sinusoïdale par un vecteur tournant (Animation disponible sur le site http://dx.doi.org/10.1051/j3ea:2006021).

A partir de ce point, l'application des vecteurs tournants à l'analyse des circuits électriques nécessite que l'étudiant maîtrise les opérations mathématiques associées à leur représentation complexe. Le dernier écran de cette leçon présente donc quelques animations illustrant les opérations mathématiques de base sur ces grandeurs (addition de deux grandeurs de même pulsation, multiplication par une constante, dérivation, intégration).

Figure 2d. Dérivation d'une grandeur sinusoïdale (Animation disponible sur le site http://dx.doi.org/10.1051/j3ea:2006021).

Electronique de Puissance : comportement dynamique d'un hacheur élévateur de tension

Les problèmes auxquels on se heurte dans l'enseignement de l'électronique de puissance sont liés à la difficulté des étudiants d'appréhender le fonctionnement en commutation inhérent aux circuits électroniques de puissance. En particulier, ils ont beaucoup de mal à comprendre qu'en fonctionnement normal, un convertisseur électronique de puissance tendra vers un comportement formé d'une succession de régimes transitoires qui se répètent de manière périodique ou quasi-périodique et à déterminer ce fonctionnement. Les animations qui permettent de visualiser de manière dynamique l'évolution temporelle des tensions et des courants constituent un outil particulièrement efficace pour acquérir une compréhension intuitive du fonctionnement de ces convertisseurs. A titre d'exemple, on peut considérer le laboratoire virtuel consacré à la simulation d'un hacheur abaisseur de tension (buck converter).

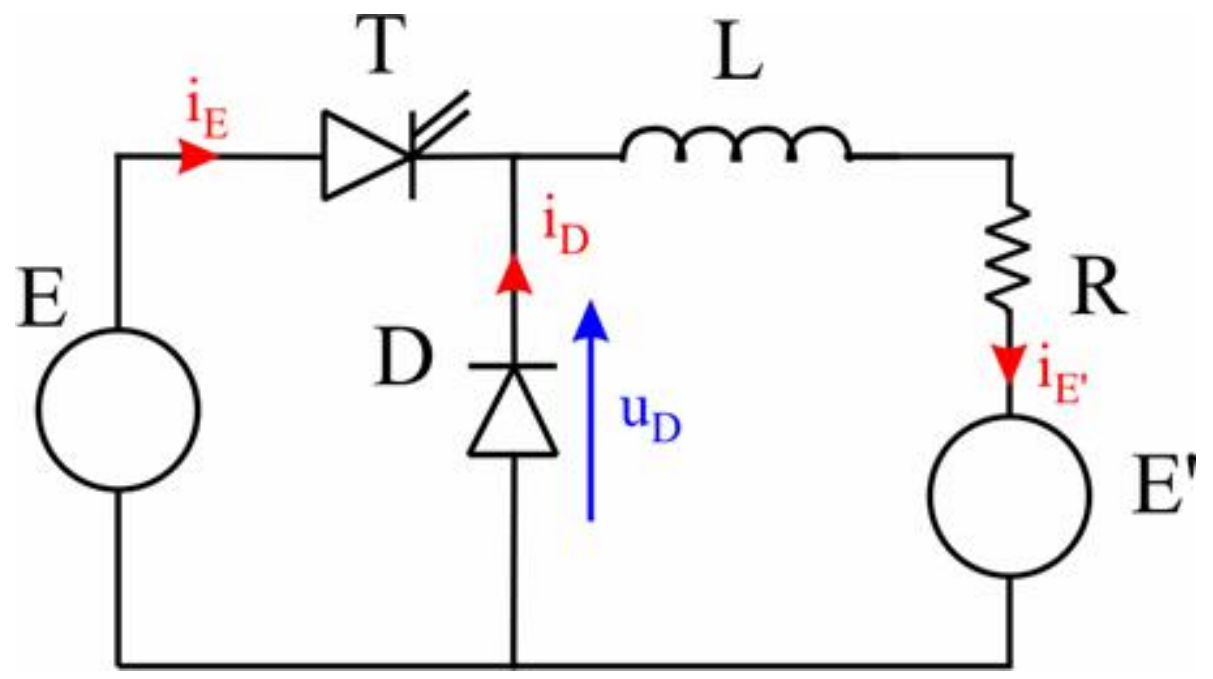

Figure 3a. Schéma du hacheur abaisseur de tension.

Lorsqu'on débute le processus de hachage avec un taux de hachage sensiblement supérieur à $E / E$ ', on tend par une succession de régimes transitoires vers un "régime permanent" pour lequel on obtient un fonctionnement cyclique à l'échelle de la période de hachage, les régimes transitoires successifs se répétant de manière identique de période en période. 


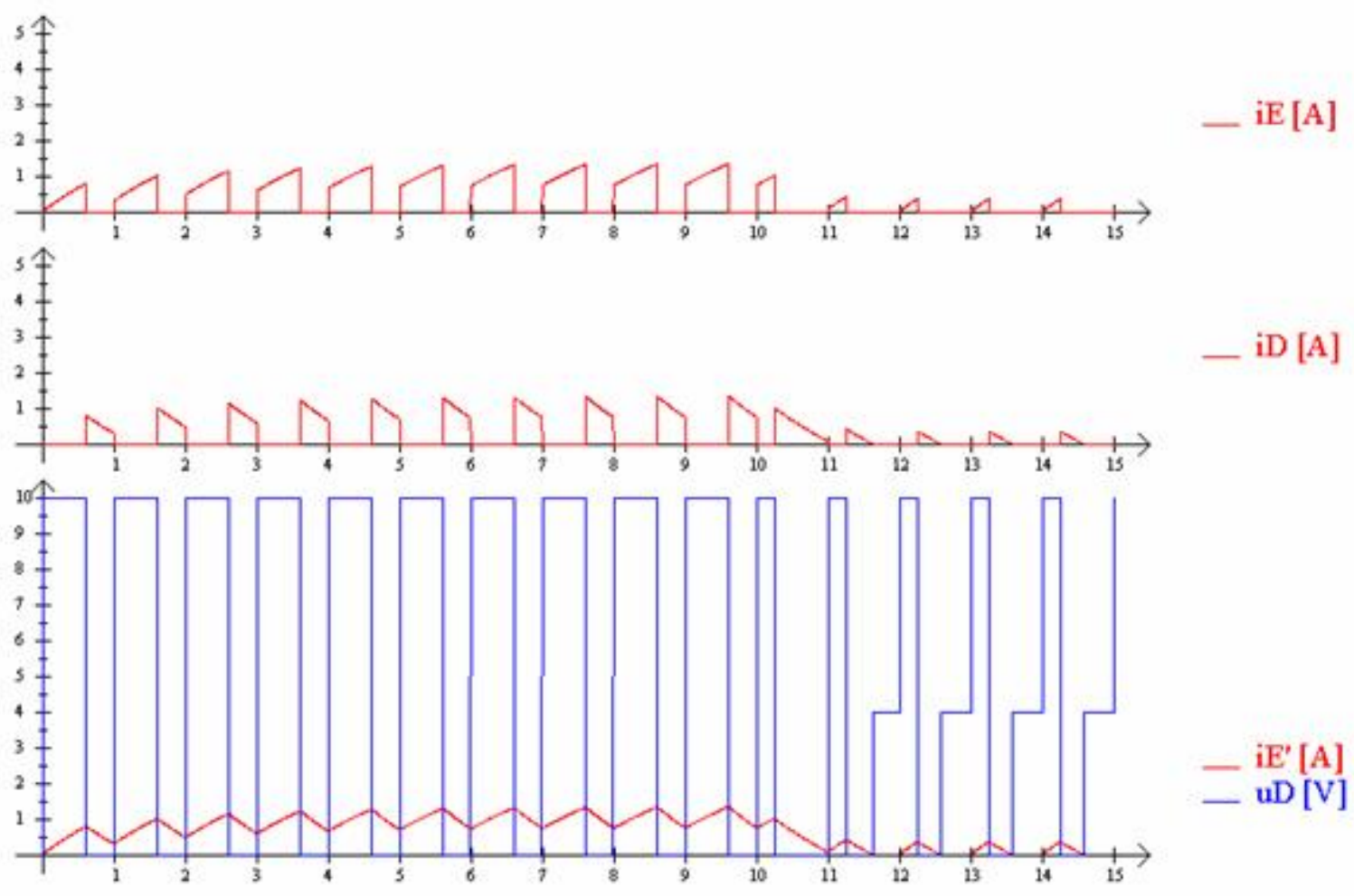

Figure 3b. Démarrage d'un hacheur série d'un passage en conduction intermittente.Si à ce moment, on diminue la valeur du temps de conduction du transistor en dessous de la valeur minimum assurant une circulation permanente du courant, on tend vers un nouveau régime ou sur chaque période de hachage, on a successivement conduction du transistor puis conduction de la diode et enfin blocage de la diode et du transistor.

\section{Machines asynchrone : construction}

Le support multimédia permet de montrer les principales pièces d'une machine asynchrone et de voir le processus de fabrication au travers de courtes séquences vidéos. Dans la leçon correspondante, une figure en coupe de la machine asynchrone permet de situer les différentes pièces (Figure 4.a) et de les voir sur la photo d'une réalisation en écorché de la machine (Figure 4.b).

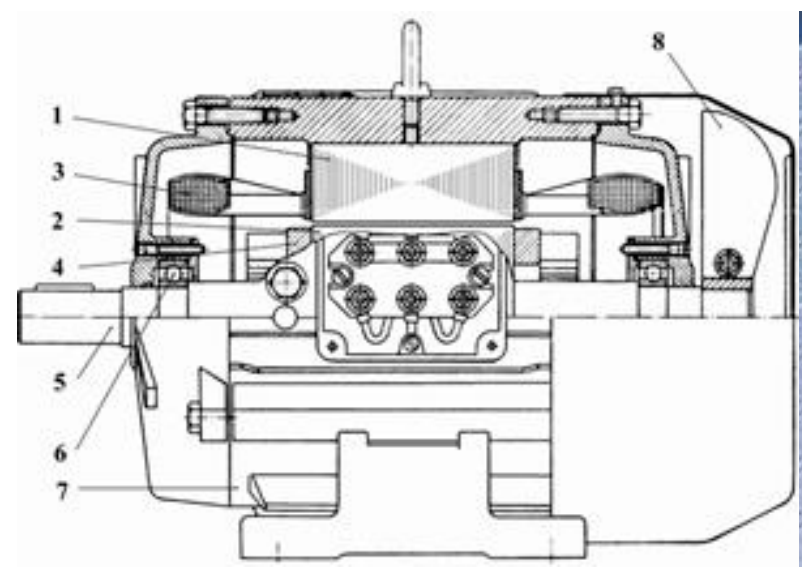

a)

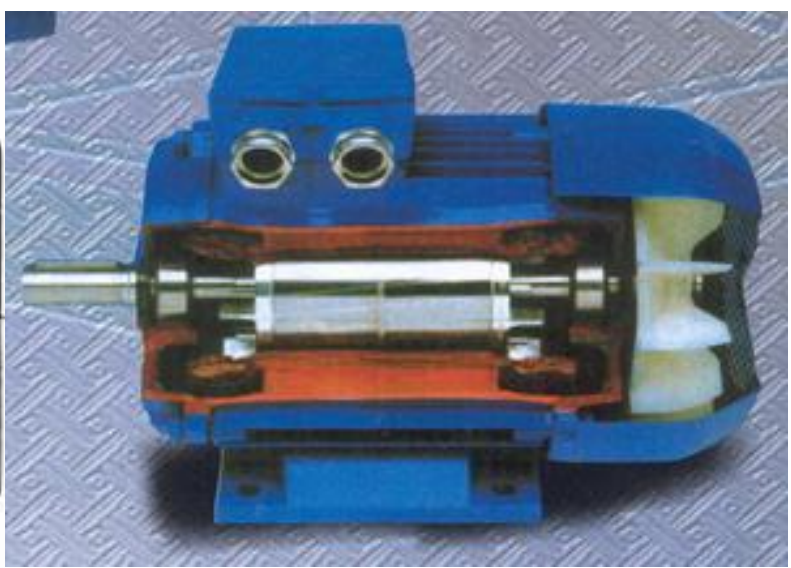

b) 
Figure 4. Vue en coupe d'une machine asynchrone

Sept vidéos illustrent ensuite les différentes étapes du processus de fabrication d'un moteur asynchrone à rotor bobiné : emboutissage des tôles et assemblage des circuits magnétiques statoriques et rotoriques, réalisation des bobinages, mise en forme, isolation, contrôle de leur résistance et insertion de ceux-ci dans les encoches du rotor et du stator. Ces vidéos ont été tournées grâce au concours de l'entreprise "Electroputere S.A", Craiova, Roumanie. A tritre d'exemple, la vidéo ci-dessous montre la réalisation d'un bobinage statorique et sa mise en forme en vue d'être inséré dans les encoches du circuit magnétique avec le pas polaire désiré.

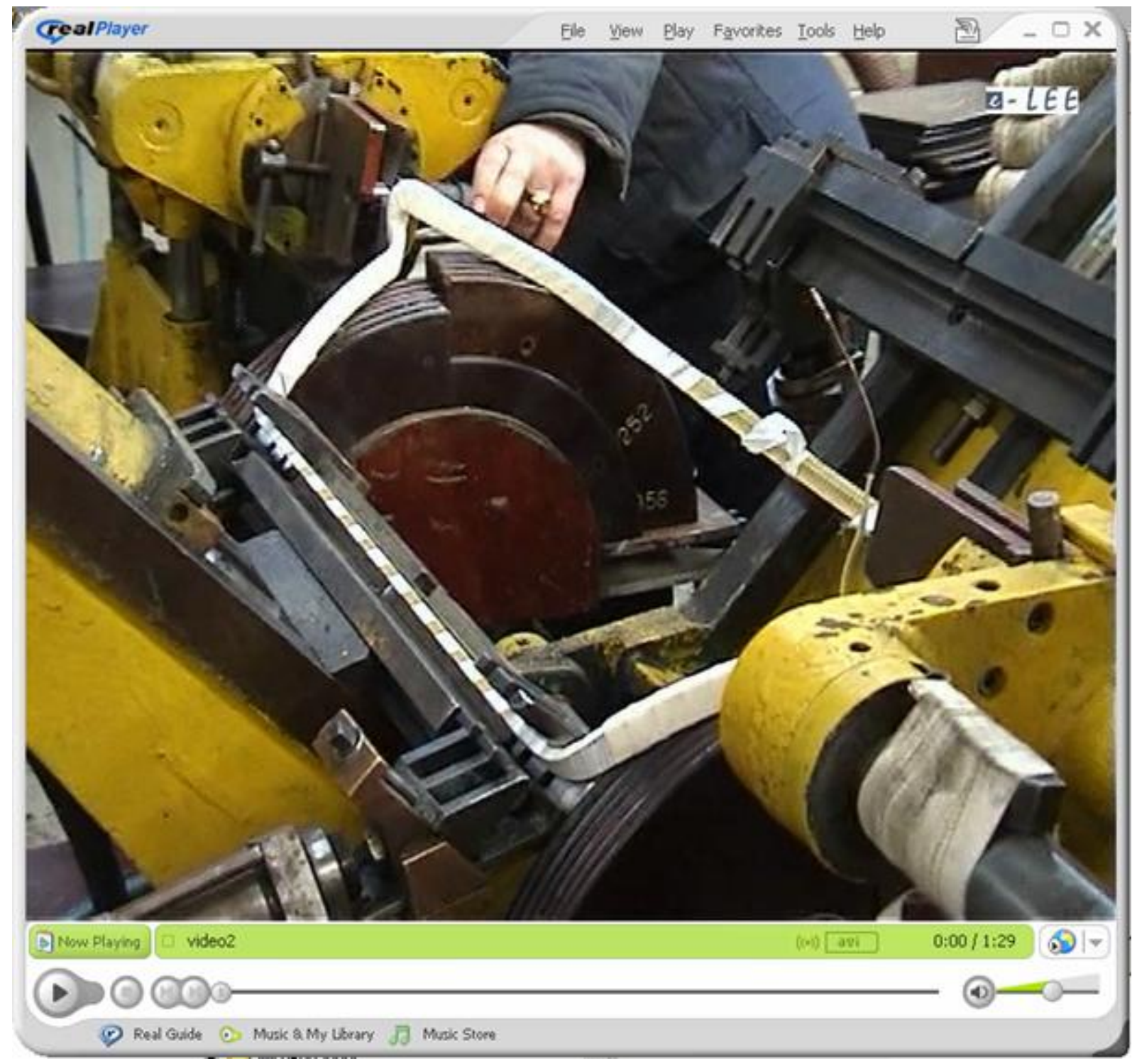

Figure 4c (Animation disponible sur le site http://dx.doi.org/10.1051/j3ea:2006021). Processus de fabrication de la machine asynchrone.

Projetées en cours, ces vidéos permettent à l'enseignant de donner toutes les explications relatives au processus de fabrication des moteurs et les contraintes qui en découlent dans la conception des moteurs. 
La notion de champ tournant est une notion essentielle pour comprendre le fonctionnement des machines asynchrones et synchrones. Expliquer comment il est possible avec trois bobinages fixes décalés de $120^{\circ}$ et alimentés par des courants déphasés également de $120^{\circ}$, de créer un champ d'entrefer (entre le rotor et le stator) équivalent à celui que produirait un seul bobinage tournant n'est cependant pas facile. La simple démonstration mathématique du théorème de Ferraris ne suffit pas toujours à convaincre les étudiants. La leçon correspondante, montre, dans un premier temps, qu'un bobinage fixe, alimenté par un courant alternatif, crée un champ pulsatoire de direction constante (la direction du champ est définie comme étant la direction pour laquelle l'amplitude du champ est maximale). Il s'agit bien ici de montrer au sens de visualiser et pas seulement de démontrer sous forme d'un calcul mathématique.

Figure 5a. Alimentation d'un bobinage fixe par un courant alternatif (Animation disponible sur le site http://dx.doi.org/10.1051/j3ea:2006021).

L’amplitude et le sens (le signe) du champ Ha créé par le bobinage $a$ varient avec ceux du courant $i a=I$.cos wt qui l'alimente :

$$
H_{a}(\theta)=K I \cos \omega t \cdot \cos \theta
$$

(w désigne la pulsation du courant, q est la position du point de l'entrefer où le champ est évalué, $K$ est une constante liée aux paramètres constructifs de la machine). Les champs créés par les trois bobinages $a, b$ et $c$ sont spatialement déphasés de $120^{\circ}$. Les courants $i a$, $i b$, ic qui les alimentent sont temporellement déphasés de $120^{\circ}$. Quand on additionne les champs produits, on obtient un champ total $\mathrm{Ht}$ :

$$
\begin{aligned}
H_{t}(\theta) & =H_{a}(\theta)+H_{b}(\theta)+H_{c}(\theta) \\
& =K I[\cos \omega t \cdot \cos \theta+\cos (\omega t-2 \pi / 3) \cdot \cos (\theta-2 \pi / 3)+\cos (\omega t+2 \pi / 3) \cdot \cos (\theta+2 \pi / 3)]
\end{aligned}
$$

Un “simple” calcul trigonométrique montre que ce champ total s’écrit encore :

$$
H_{t}(\theta)=\frac{3}{2} K I \cos (\theta-\omega t)
$$

Ce résultat peut également s’établir également graphiquement :

Figure 5b. Champ tournant (Animation disponible sur le site http://dx.doi.org/10.1051/j3ea:2006021).

La somme des trois vecteurs représentatifs du champ magnétique est un vecteur d'amplitude constante mais dont la direction varie avec le temps_. C'est ce qu'on appelle un champ tournant.

Si on place un aimant permanent dans ce champ tournant, il suivra ce champ entraînant le rotor de la machine dans un mouvement de rotation (principe de fonctionnement des moteurs synchrones). Si on place un matériau conducteur au sein de ce champ, il sera le siège de 
courants induits dont on peut montrer, en vertu de la loi de Lenz, que les forces de Laplace qu'ils créent en interaction avec le champ magnétique sont telles qu'elles entraînent le rotor dans un mouvement de rotation à une vitesse nécessairement légèrement inférieure à celle du champ tournant (principe de fonctionnement des moteurs asynchrones). Le recours à l'animation graphique développe chez l'étudiant une compréhension intuitive du fonctionnement des machines électriques sur laquelle l'enseignant peut se reposer pour la suite de son cours.

\section{Machines asynchrones : Direct Torque Control}

Le recours à l'animation permet également de faire comprendre des notions avancées comme la commande directe en couple (Direct Torque Control - DTC) du moteur asynchrone. Cette stratégie de commande qui s'énonce davantage sous forme de règles qu'au travers un algorithme mathématique n'est pas toujours aisée à comprendre, tant qu'on ne possède pas une connaissance suffisante du fonctionnement interne de la machine asynchrone. Tout d'abord on montre que pour maintenir un état magnétique aussi constant que possible de la machine asynchrone (et avoir un modèle de la machine où l'on peut faire abstraction du phénomène de saturation magnétique), il convient de conserver autant que possible l'amplitude du flux statorique constante. La trajectoire de l'extrémité du vecteur représentatif de ce flux statorique doit donc être proche d'un cercle (figure 6a)

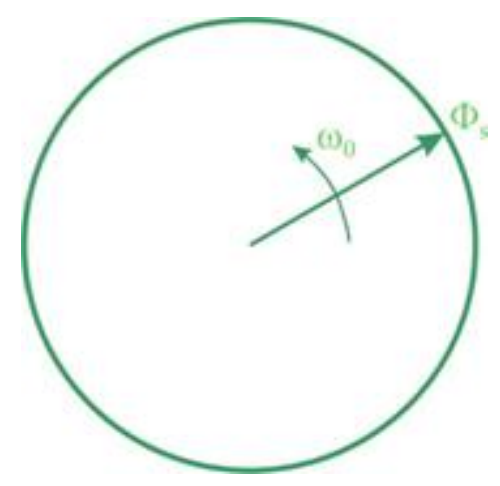

Figure 6a. Trajectoire du vecteur représentatif du flux statorique.

On établit ensuite que le couple de la machine asynchrone peut être contrôlé en réglant la vitesse de rotation de ce vecteur. En l'accélérant, on augmente la valeur du couple (figure 6.b), en le ralentissant on réduit cette valeur, voire on obtient un couple de freinage (couple négatif - figure 6.c).

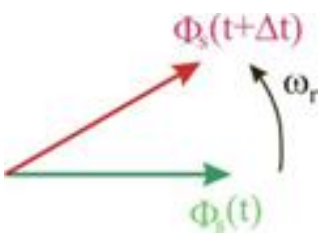

b)

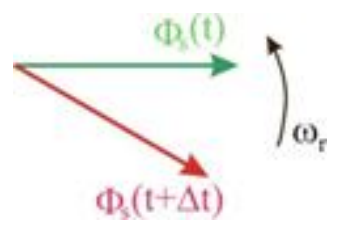

c)

Figure 6. Réglage de la vitesse d’avance du flux statorique. 
La position actuelle du vecteur représentatif du flux statorique peut être estimée à partir de la mesure des courants et tensions au stator. Sa position au prochain pas de temps dépendra de la valeur du vecteur des tensions qui sera appliquée d'ici là. Faisant le lien avec la thématique "électronique de puissance », on montre qu'avec un onduleur de tension, 7 valeurs différentes peuvent être appliquées à un instant $t$ (figure 6d).

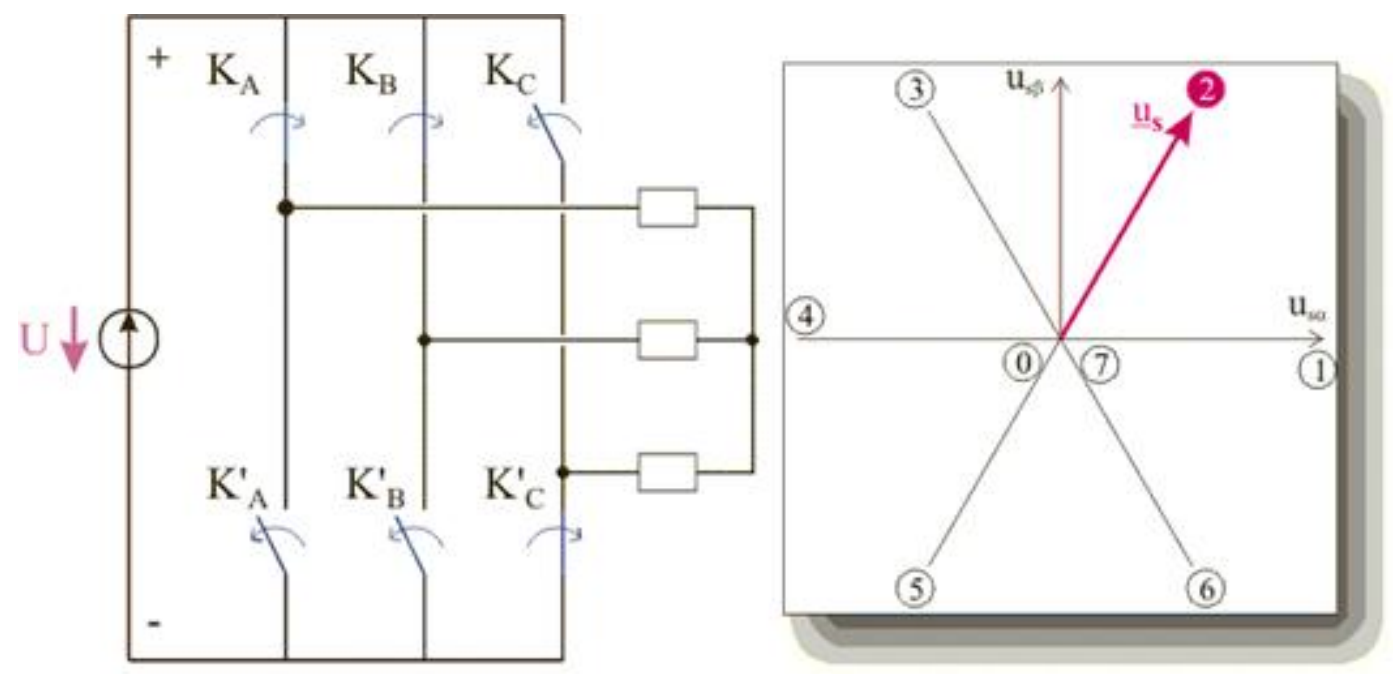

Figure 6d. Vecteur des différentes tensions applicables et topologies correspondantes de l'onduleur de tension

Le vecteur des tensions statoriques est, au cours de la période de temps où celui ci est appliqué et en première approximation, l'image de la direction dans laquelle évoluera le vecteur des flux statoriques. Fonction de l'amplitude (inférieure ou supérieure à sa référence), de la direction actuelle de ce vecteur (le secteur qu'il occupe), et de la direction que l'on souhaite lui voir prendre par la suite (fonction du couple souhaité), l'un ou l'autre de ces 7 vecteurs de tensions sera appliqué.

Figure 6e. Contrôle du flux statorique (Animation disponible sur le site http://dx.doi.org/10.1051/j3ea:2006021)

Energies renouvelables: recherche du point de puissance maximale d'une installation photovoltaïque

Les outils actuellement développés dans la thématique «Énergies renouvelables » du site concernent les filières éolienne et solaire photovoltaïque. Les TP virtuels y trouvent plus qu'ailleurs leur place du fait de la difficulté voire l'impossibilité de réaliser, à l'échelle de puissance représentative des systèmes réels, des manipulations sur des systèmes éolien et solaire photovoltaïque. Une des originalités de cette partie du site est que la définition et la réalisation des ressources sont principalement le fait d'étudiants ingénieurs de dernière année, supervisés bien entendu par leurs professeurs qui en ont validé le contenu scientifique. Ces étudiants y ont apporté leur propre perception des aspects difficiles à comprendre de la matière, ou des points à mettre en avant pour rendre le site attractif et motivant. Une autre originalité des développements sur la thématique "énergies renouvelables » est qu'ils s’intègrent dans un site traitant de différents aspects de l'électrotechnique qui sont largement utilisés dans les sources à énergie renouvelable. 
La figure 7a illustre une animation interactive des principaux constituants d'une éolienne.

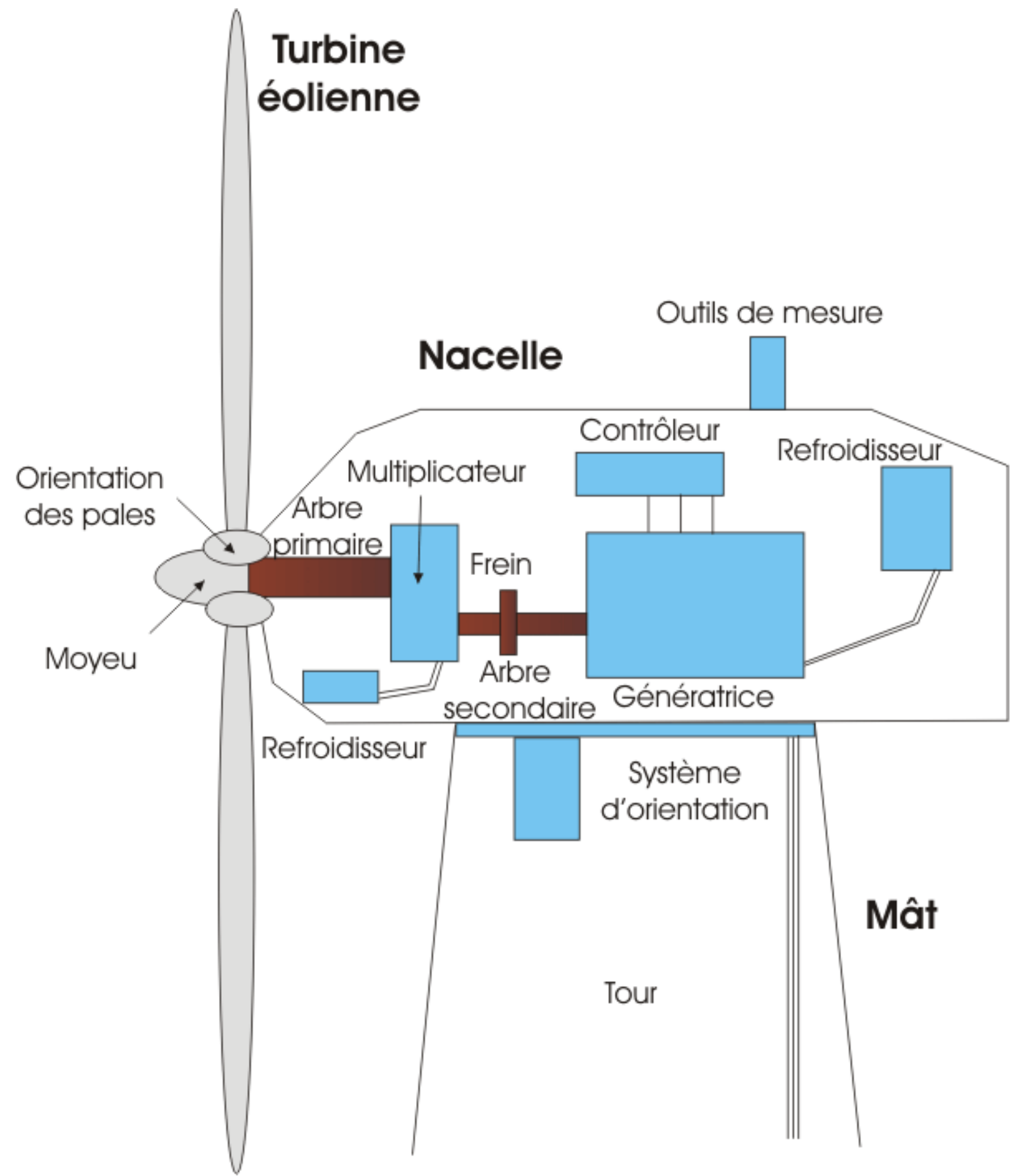

Figure 7a. Animation permettant de découvrir les différents constituants d'une éolienne en cliquant sur ces différents composants.

La puissance générée par une éolienne à vitesse variable particulière (basée sur une machine asynchrone contrôlée via les bobinages rotoriques), en fonction de la vitesse du vent et de l'angle d'orientation des pales, est illustrée par une animation interactive présentée à la figure $7 b$ : 
Figure 7b. Transfert de puissance dans une éolienne à vitesse variable (Animation disponible sur le site http://dx.doi.org/10.1051/j3ea:2006021)

Les générateurs photovoltaïques sont également présentés à l'aide d'animations interactives permettant d'illustrer, par exemple, l'influence de l'éclairement, de la température et du maximum power point tracking (MPPT) permettant de maximiser la puissance générée par les cellules photovoltaïques (figure 7c).

Figure 7c. Variation du point de puissance maximale dans le plan courant-tension en fonction de la température d'un panneau photovoltaïque. (Animation disponible sur le site http://dx.doi.org/10.1051/j3ea:2006021)

Des animations interactives permettent un dimensionnement (à vocation uniquement pédagogique) de panneaux photovoltaïques alimentant une maison isolée ou connectés au réseau de distribution de l'électricité en intégrant les besoins en terme de consommation.

Le fait que l'amplitude du champ résultant est effectivement maximale dans la direction donnée par la somme des vecteurs représentatifs des champs créés par chacun des trois bobinages n'est pas démontrée mathématiquement dans le site. Ce résultat est cependant intuitivement admis par les étudiants : aucune question ne nous a été posée à ce sujet.

\section{Développement d'une "e-learning toolbox"}

La réalisation de telles ressources multimédia, en particulier celles présentant une interactivité élevée nécessite la mobilisation d'une force de travail considérable. De la première esquisse d'un projet, née de l'imagination d'un enseignant désireux d'exploiter le potentiel des TIC pour expliquer au mieux un concept physique à la finalisation de la ressource correspondante, le chemin est long, laborieux et semé de difficultés techniques :

1. scénarisation de la ressource (écriture du texte, découpage des pages, définition des animations) 


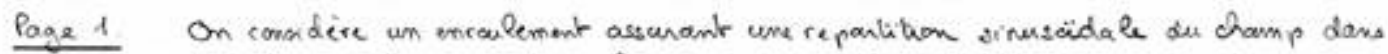

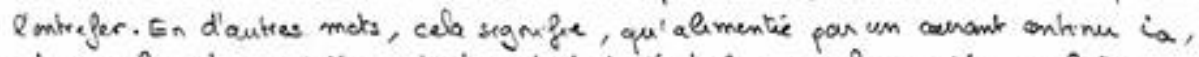

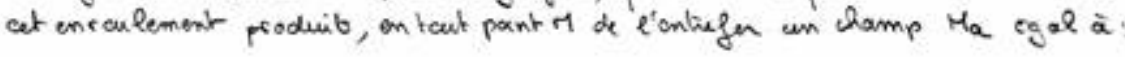

$H_{a}=K_{1} \cdot i_{a} \cdot \cos \theta$

ai Oost la protum de paint $M$
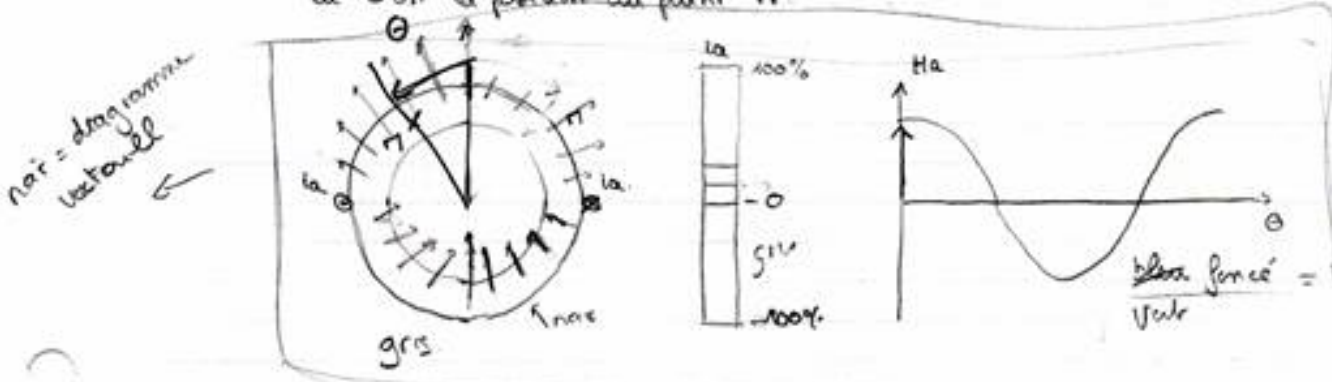

seagreen

Navey

bers. foncé $=$ Nonglibise

Figure 8a. Le travail de scénarisation d'une ressource pédagogique. 
2. saisie informatique du texte et des équations

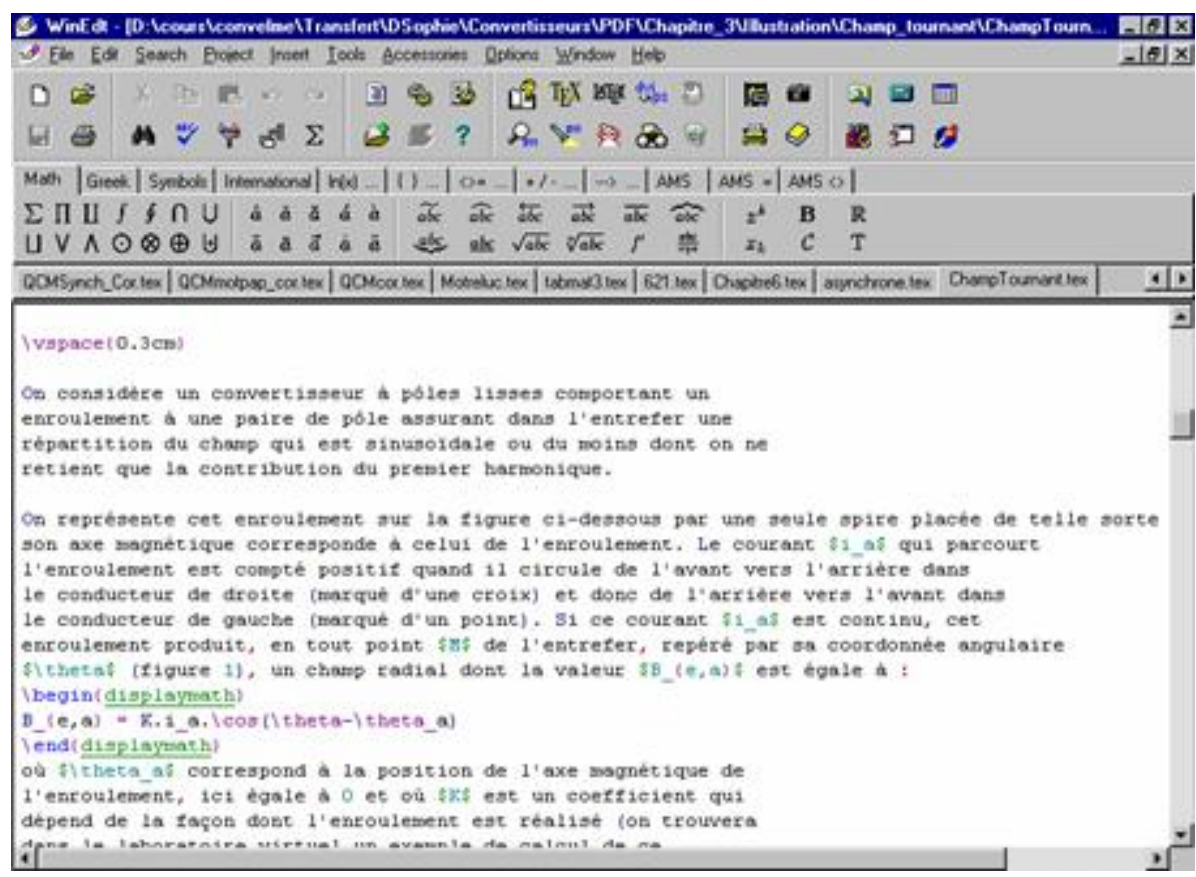

Figure 8b. La saisie informatique du texte et des équations.

3. transfert sous format HTML et traitement spécifique des équations (génération de fichiers images)

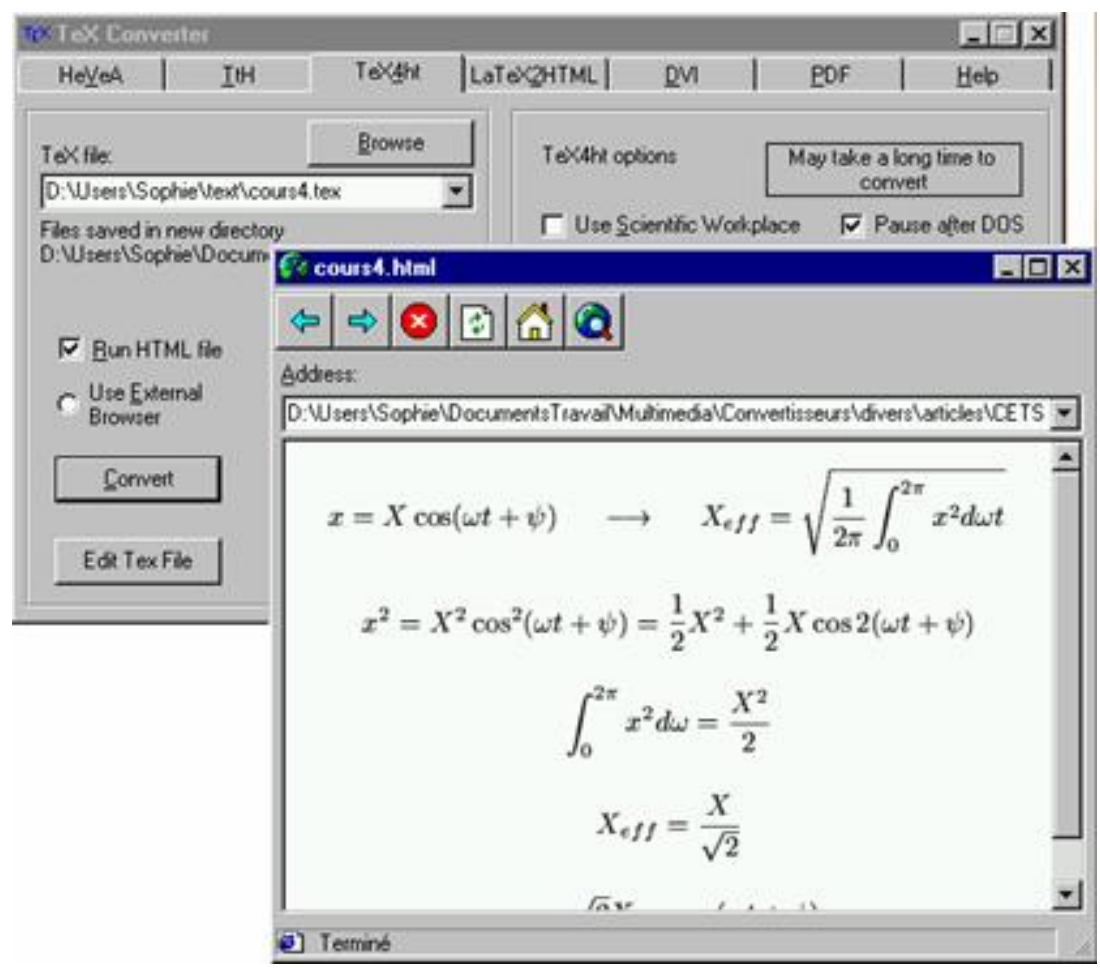

Figure 8c. Génération des équations sous format "gif". 
4. création des figures, prises de vue (photos et vidéos)

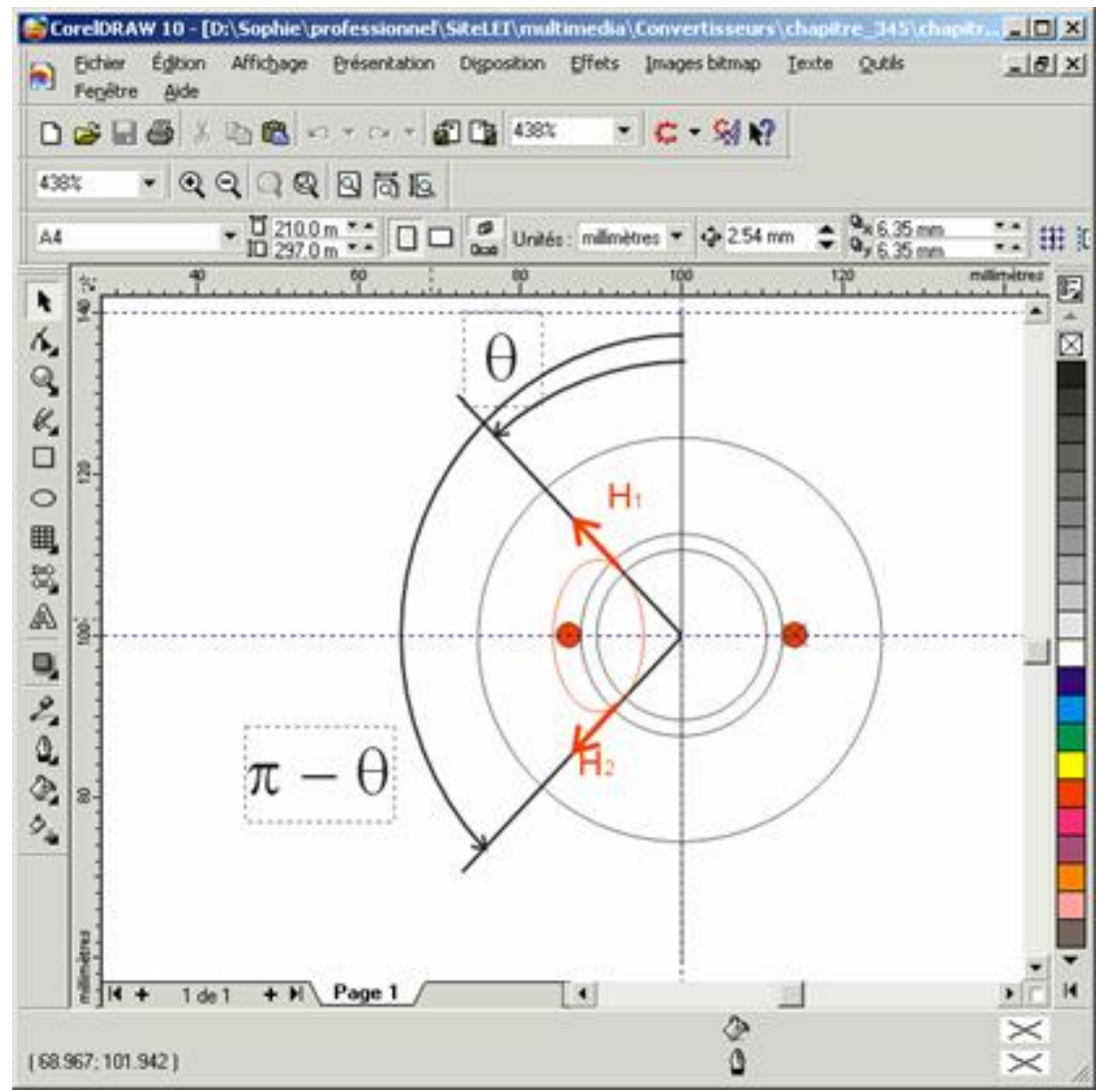

Figure 8d. Création des figures.

5. création d'une version imprimable (sous format PDF par exemple)

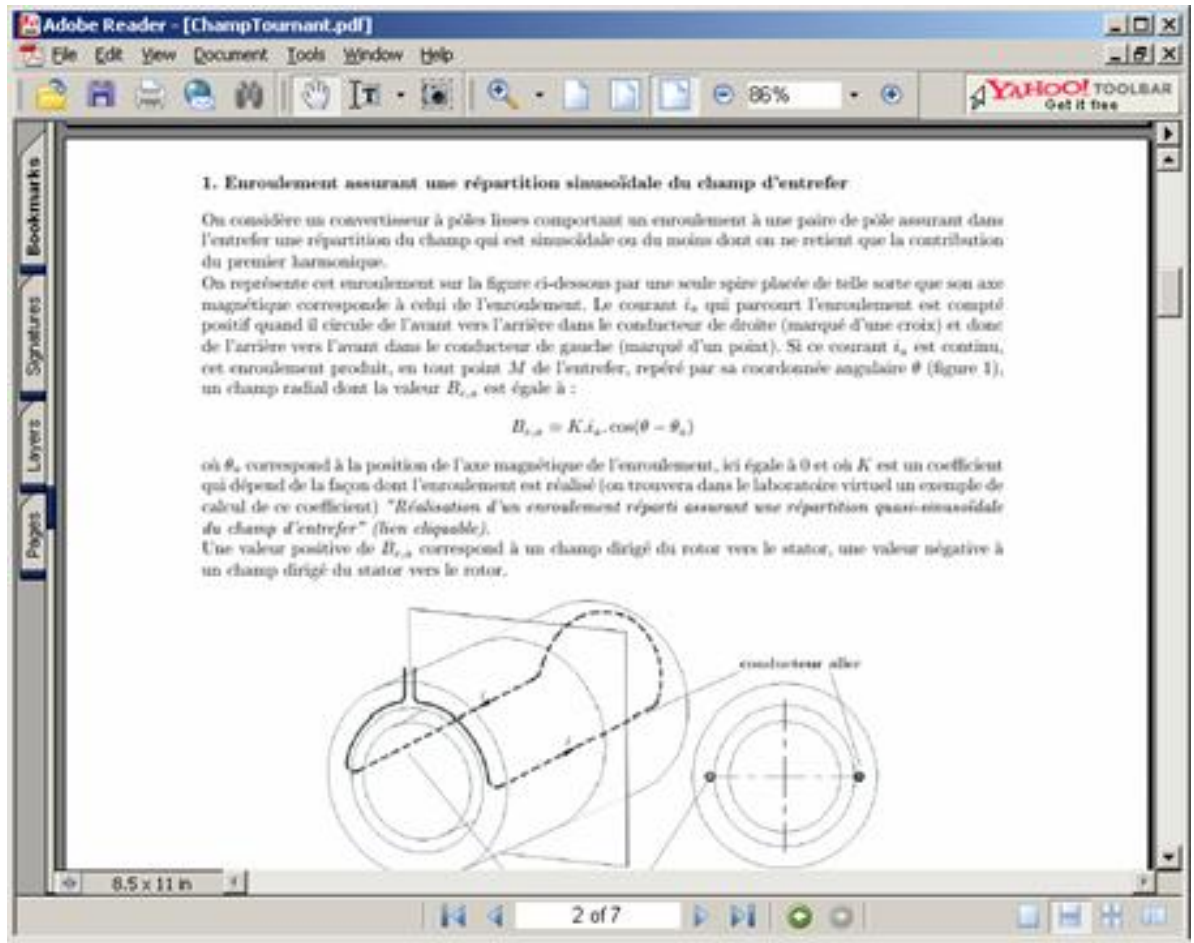

Figure 8e. Création d'une version imprimable. 


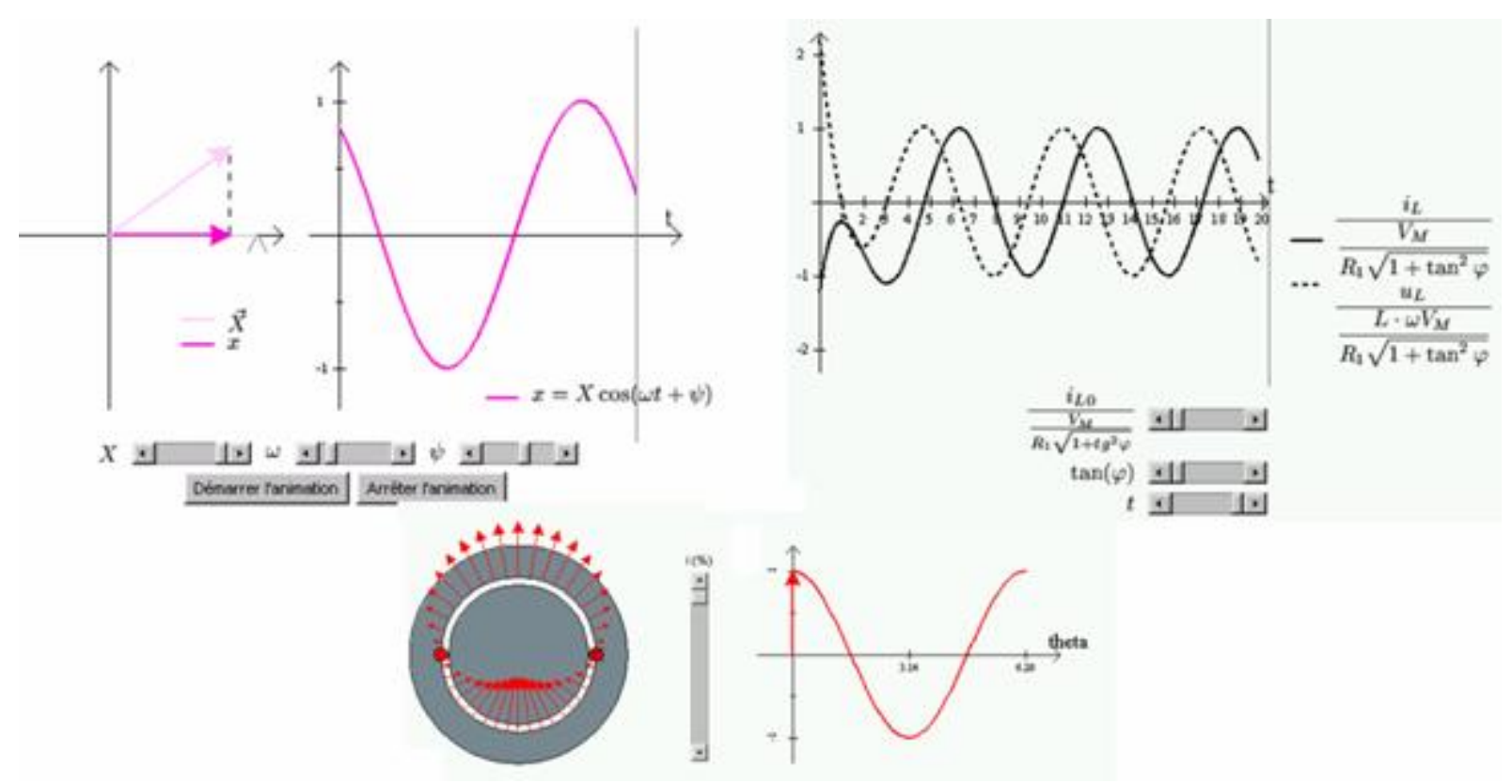

Figure 8e. Programmation des animations.

7. finalisation de la page, gestion de la navigation entre les pages et leur intégration dans le site.

La réalisation de chacune de ces étapes suppose la maîtrise de méthodes et outils informatiques spécifiques. Une première difficulté rencontrée lors du projet "e-LEE" était d'apporter un support technique aux enseignants partenaires du projet, qui ne disposaient pas forcément de compétences pointues en informatique.

La coordination du travail entre plusieurs équipes d'enseignants et de développeurs et l'intégration des ressources en un site unique et cohérent constituait un défi supplémentaire. Il paraissait en effet indispensable de retrouver d'une ressource à l'autre un style graphique qui donne l'impression d'un site unique et non d'une multiplicité de réalisations disparates. Mais davantage que cela, il fallait organiser la navigation au sein du site de façon cohérente, efficace et conviviale, tout en prévoyant les possibilités d'évolution du site.

L'approche proposée a été celle de la constitution et du partage d'une "e-learning toolbox" en vue de faciliter pour les enseignants le développement de leurs propres ressources. Le travail en coopération a permis à chaque partenaire de s'investir dans la prise en main approfondie de certains de ces outils et de se contenter, pour le reste, des outils d'aide proposés par d'autres équipes de développeurs.

L'utilisation des ces outils doit permettre la réalisation des tâches les plus basiques en suivant à la lettre les instructions fournies (fiches de documentation). Cette approche, à savoir permettre à des personnes qui ne sont pas forcément des spécialistes informatiques de réaliser par eux-mêmes l'essentiel des ressources multimédia, a été validée en confiant cette réalisation à des étudiants de génie électrique de HEI effectuant un travail de fin d'études. 
La plupart néophytes en programmation Java, ils se sont, en quelques mois, montrés capables de réaliser des ressources pédagogique comprenant des applets interactives et s’intégrant dans le site.

La "e-learning toolbox" a donc été conçue et développée en vue de faciliter le développement par les enseignants de leurs propres ressources pédagogiques et leur intégration sur le site. Cette section détaille l'ensemble des méthodes et outils informatiques que constitue cette boîte à outils d'aide à la conception de ressources.

\section{Modèles de pages}

La nécessité de définir une charte graphique et des principes de navigation cohérents à travers tout le site est rapidement apparue :

1. la définition et le respect d'une charte graphique permet de conserver une certaine unité à travers toutes les pages du site, quelle que soit l'équipe qui en a assuré le développement ;

2. un modèle de navigation cohérent a été défini, organisé sur 4 niveaux : thématiques, chapitres, éventuellement subdivisés en sections et enfin les ressources elles-mêmes (exposés, laboratoires, exercices, qcm). Seule la navigation au sein même d'une ressource est laissée à la libre appréciation de son concepteur. De la sorte, l'internaute retrouve une structure identique à travers les différentes thématiques développées, qui lui évite le sentiment de "se perdre" à travers les pages du site. 


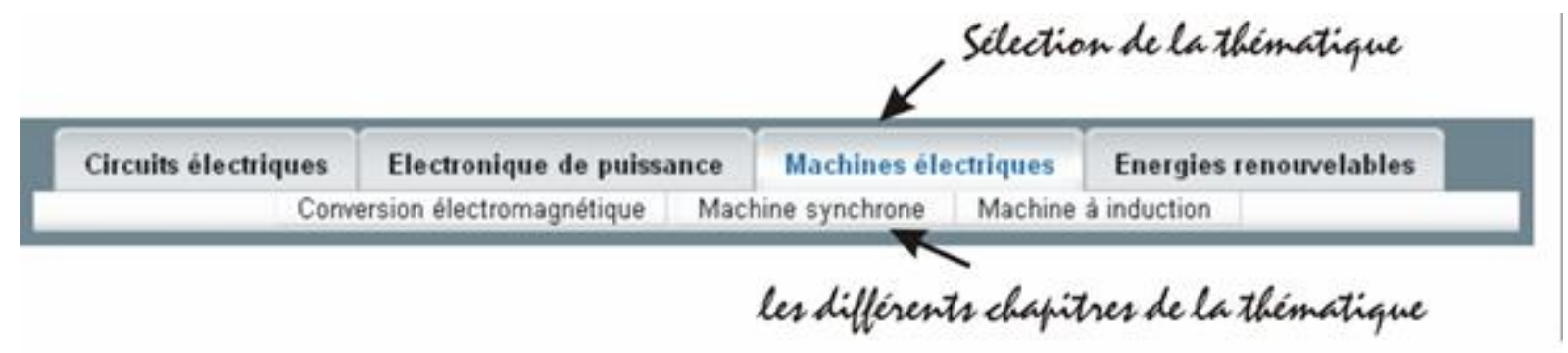

\section{titre}

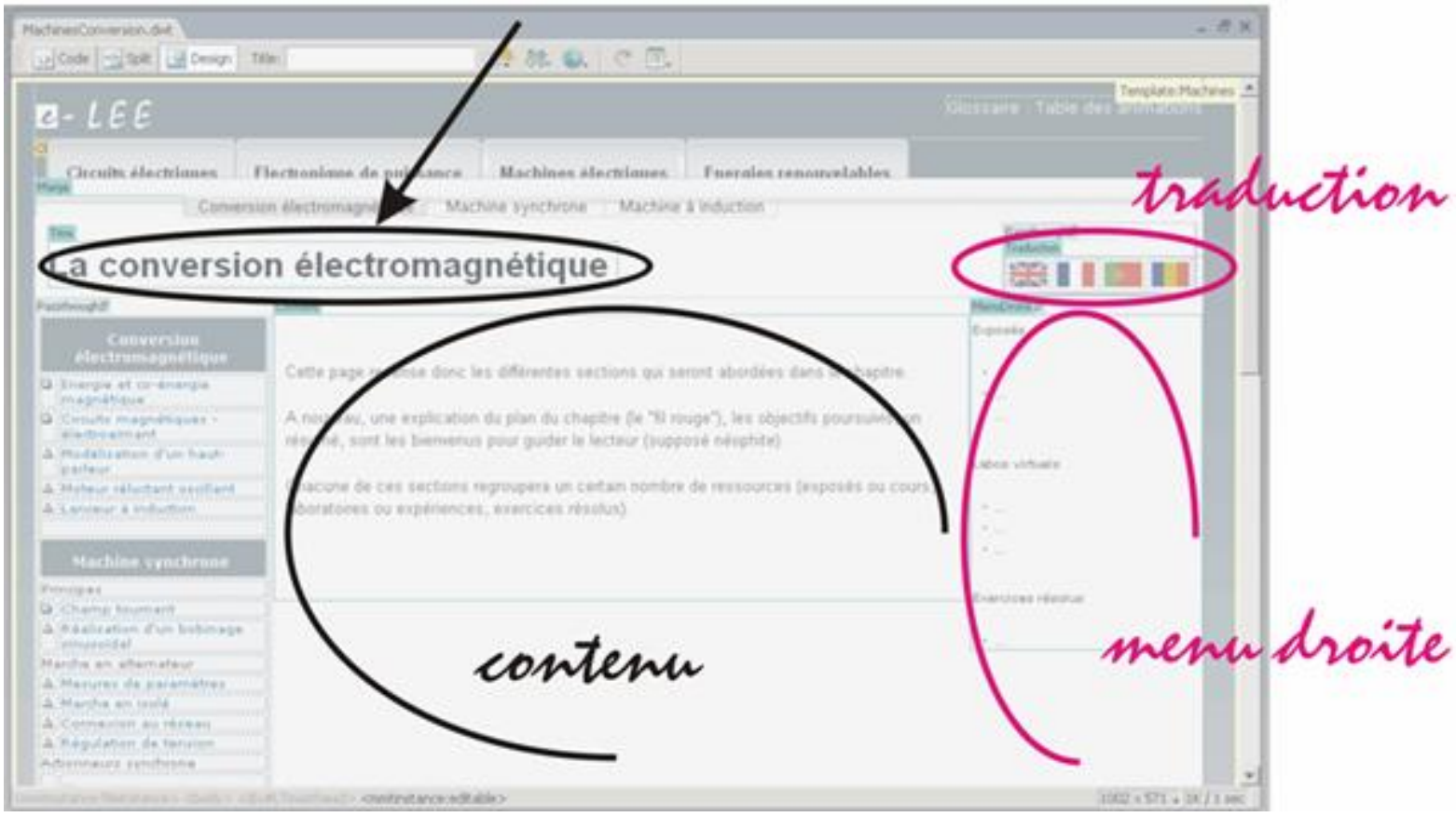

Figure 9. Définition des principes de navigation dans le site.

Cette charte et ces principes de navigation sont mis en œuvre concrètement par l'application de "Templates" ou "Modèles de page" proposé par le logiciel Dreamweaver (utilisé pour la création de pages Web). Il s'agit d'un type spécial de document qui sert à concevoir une mise en page "fixe". Certaines zones sont définies comme modifiables dans les documents basés sur ce modèle. Le reste de la page, en l'occurrence les menus de navigation à travers le site et la définition d'une "feuille de style" qui gère la mise en forme des pages (polices, etc.) ne peuvent être modifiés par les différents développeurs. Seul le titre de la page, son contenu et la navigation "interne" à travers les pages d'une ressource sont modifiables. 


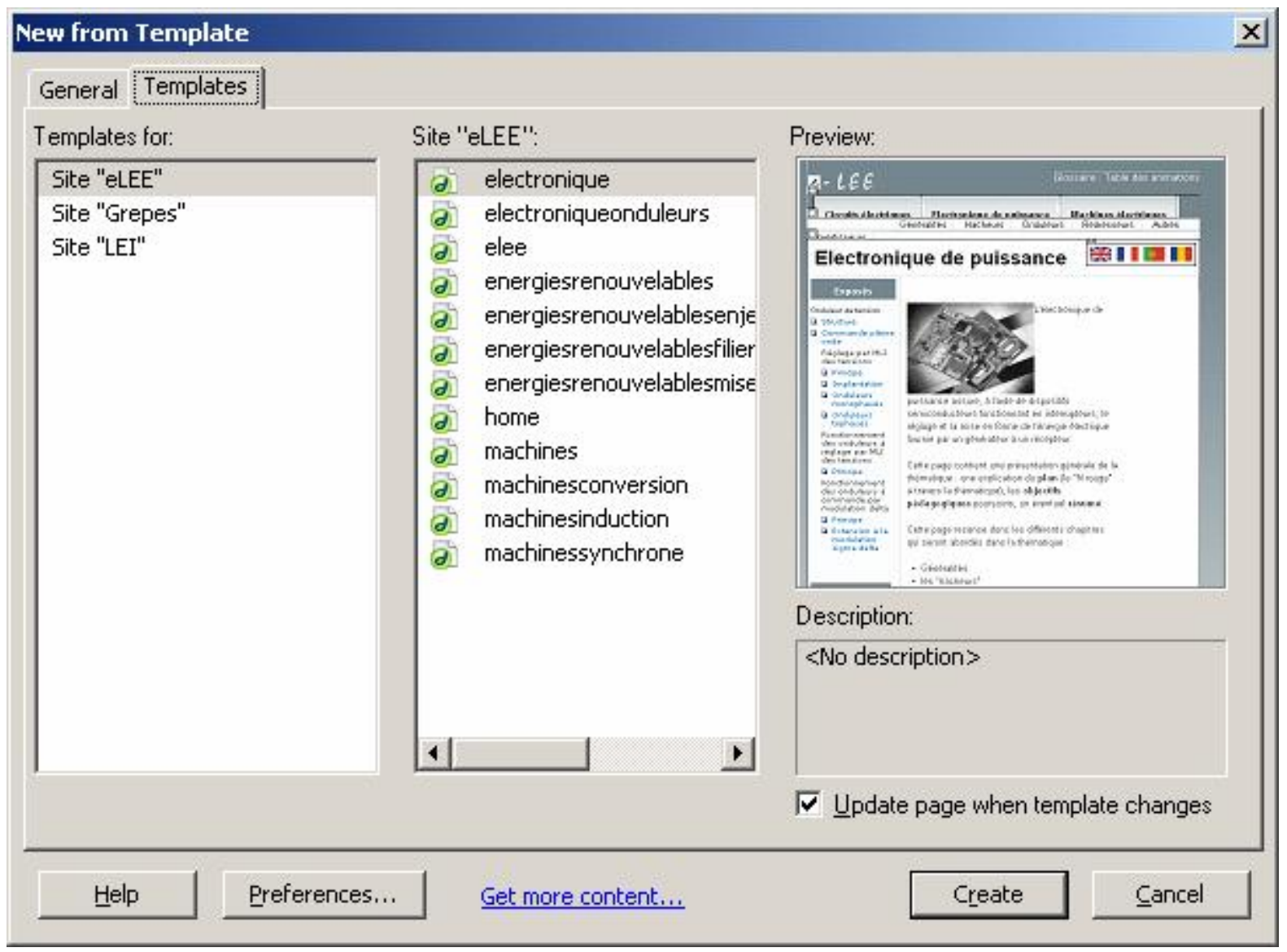

Figure 10a. Création de modèles de page. 


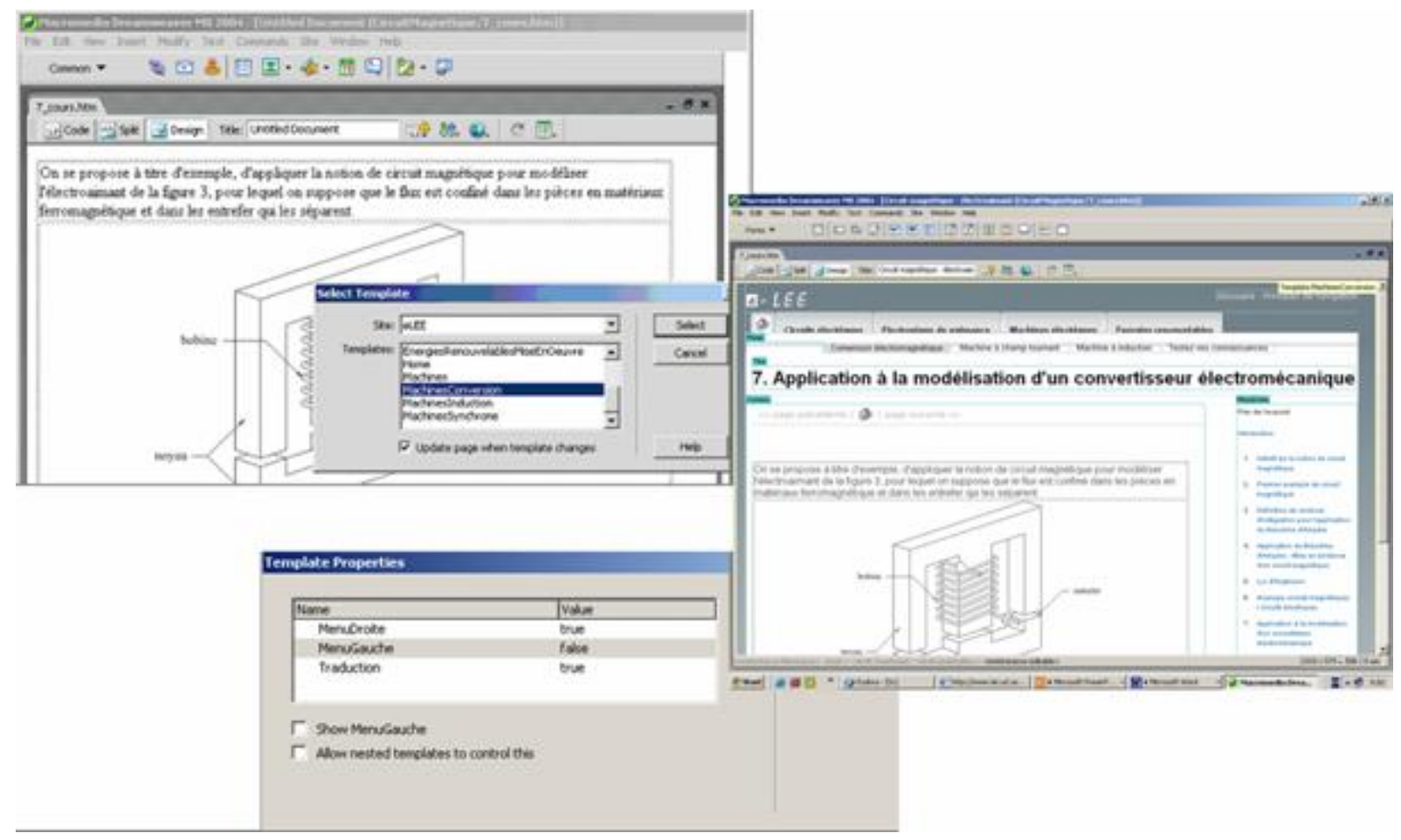

Figure 10b. Application d'un modèle de page.

Moteur de recherche

Google offre gratuitement aux institutions d'éducation et aux organisations sans but lucratif de disposer d'un moteur de recherche à travers leur propre site. L'inscription du domaine "www.e-lee.net" a permis de configurer un moteur de recherche performant à travers les pages du site, qui propose aux internautes le confort mais surtout l'efficacité des recherches Google. En moins d'une seconde s'affiche une liste de pages correspondantes à la requête encodée par l'internaute. Cette recherche se fait, au choix de l'utilisateur, uniquement dans le domaine www.e-lee.net ou à travers tout l'internet. L'indexation du contenu du site est géré par Google, et mis à jour environ une fois par mois. Un lien permet d'accéder depuis chaque page du site à ce moteur de recherche. Ce lien est automatiquement généré lors de l'application des modèles de pages (cfr § 5.1) sans que le développeur de la page doive configurer quoi que ce soit. 


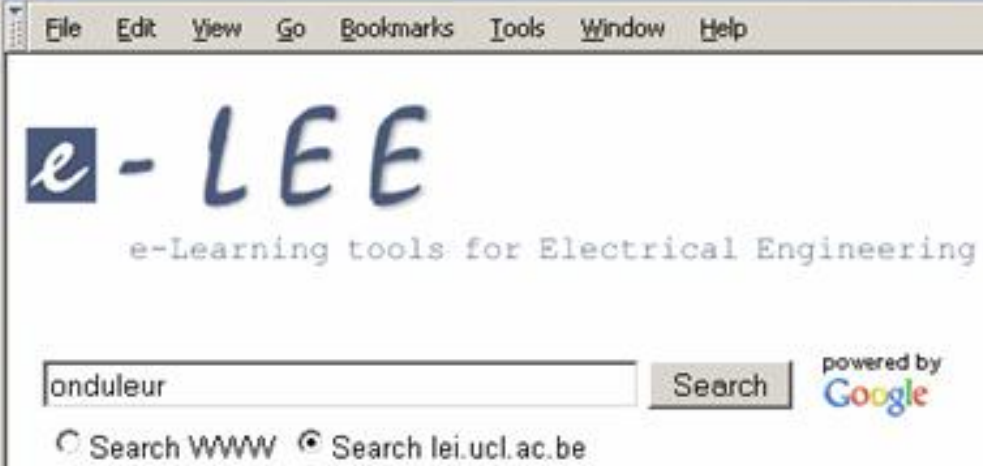

Web Searched pages from lei.ucl.ac.be for onduleur. ( 0.18 seconds)

\section{Onduleurs}

Un onduleur de tension est alimenté par un générateur à courant continu du type ... Pour respecter la règle de l'alternance des sources, l'onduleur doit ...

mmw lei.ucl ac. be/multimedia/eLEE/FR/realisations/ElectroniquePuissance/Onduleurs/Onduleurs. htm - 23k -

Cached - Similar pages

\section{Commande pleine onde}

Etude du débit sur charge RL d'un onduleur monophasé ... Ce cours montre comment un onduleur peut créer une ou des tension(s) alternative(s) en faisant ...

mww lei.ucl. ac. be/multimedia/eLEE/FR/realisations/ElectroniquePuissance/Onduleurs/CdePIOnde/C dePIOnde.htm

$-21 k$ - Cached - Similar pages

\section{Débit sur charge RL de l'onduleur monophasé}

Etude du débit sur charge RL d'un onduleur monophasé ... On détermine le courant foumi par la source qui alimente l'onduleur. Figure 1 ...

unw. lei.ucl. ac. be/.../realisations/ElectroniquePuissance/

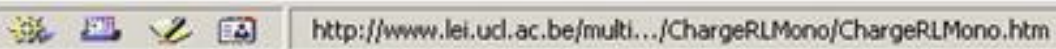

Figure 11. Le moteur de recherche sur le site "www.e-lee.net". 
Une application (en langage XML) a été programmée pour permettre à un enseignant de développer des modules d'auto-évaluation sous forme de questionnaires à choix multiple (QCM). Cette application se différencie des logiciels présents sur le marché car elle gère des problèmes propres à la publication scientifique et permet l'intégration au sein de ces QCM de graphes, d'équations, de figures, etc.

Le XML (eXtensible Markup Language) est une sorte de langage HTML amélioré, qui permet de définir de nouvelles balises.

L'application que nous avons développée pour la génération de QCM fait appel à plusieurs fichiers, dont :

1. une "grammaire", qui définit les balises pouvant être utilisées, ainsi que leurs attributs et l'imbrication des éléments possibles

2. des fichiers de traitement de l'information contenue dans le QCM.

Le contenu de ces fichiers ne change pas.

Le professeur qui souhaite développer un QCM doit créer un fichier ".xml" qui répond aux règles définies dans la grammaire. Le contenu de ce fichier est constitué d'une hiérarchie de balises comportant des attributs. Par exemple, la définition d'une question se définit par la balise <question>. On peut définir des questions à choix multiple, avec une seule ou plusieurs bonnes réponses possibles. Un deuxième attribut permet de définir la langue dans laquelle est rédigée la question. Il est possible d'intégrer des images (figures et/ou équations) dans l'intitulé de la question et des réponses. Les balises <reponse> se placent dans une balise <question>. L'attribution des valeurs "true" ou "false" à l'attribut "value" permet de définir les bonnes et les mauvaises réponses. L'enseignant a la possibilité de pondérer les questions pour le calcul du score final.

L'enseignant doit également créer deux pages HTML (l'une pour le QCM et l'autre pour le corrigé). Ces pages contiennent seulement l'appel à la fonction javascript qui permet la traduction du XML en HTML. Seuls deux paramètres doivent être précisés par l'enseignant : la langue dans laquelle afficher le questionnaire et le choix d'un calcul du score avec ou sans malus (points négatifs ou non dans le cas de réponse erronée).

L'internaute visualise la page du questionnaire, répond aux questions et valide le QCM. La page de correction se charge alors automatiquement. Elle permet à l'étudiant de visualiser sa/ses réponses (bonnes ou mauvaises), la/les bonnes réponses, le calcul de son score et lui donne accès à une explication de la réponse (si prévue par l'enseignant). 
2: Compléter la phrase :

1 : Le couple êlectromagnétique est égal à

a. $6 . .$. la dérivée partielle

b. $O$... l'opposé de la dérivée partielle

c. $C$ Je ne sais pas

2:... de la co-énergie magnétique par rapport

a. $\odot$... autemps

b. $\bigcirc$... a la position angulaire du rotor

c. $O$ Je ne sais pas

3:... si cette énergie est exprimée en fonction

a. $C$... des flux encerclés par les enroulements.

b. C... des courants circulants dans les enroulements.

c. C Je ne sais pas

3: Dans un convertisseur électromagnétique ne comportant pas d'aimants permanents et où les relations fluxcourant sont linéaires, les valeurs de l'énergie et de la co-énergie magnétique.

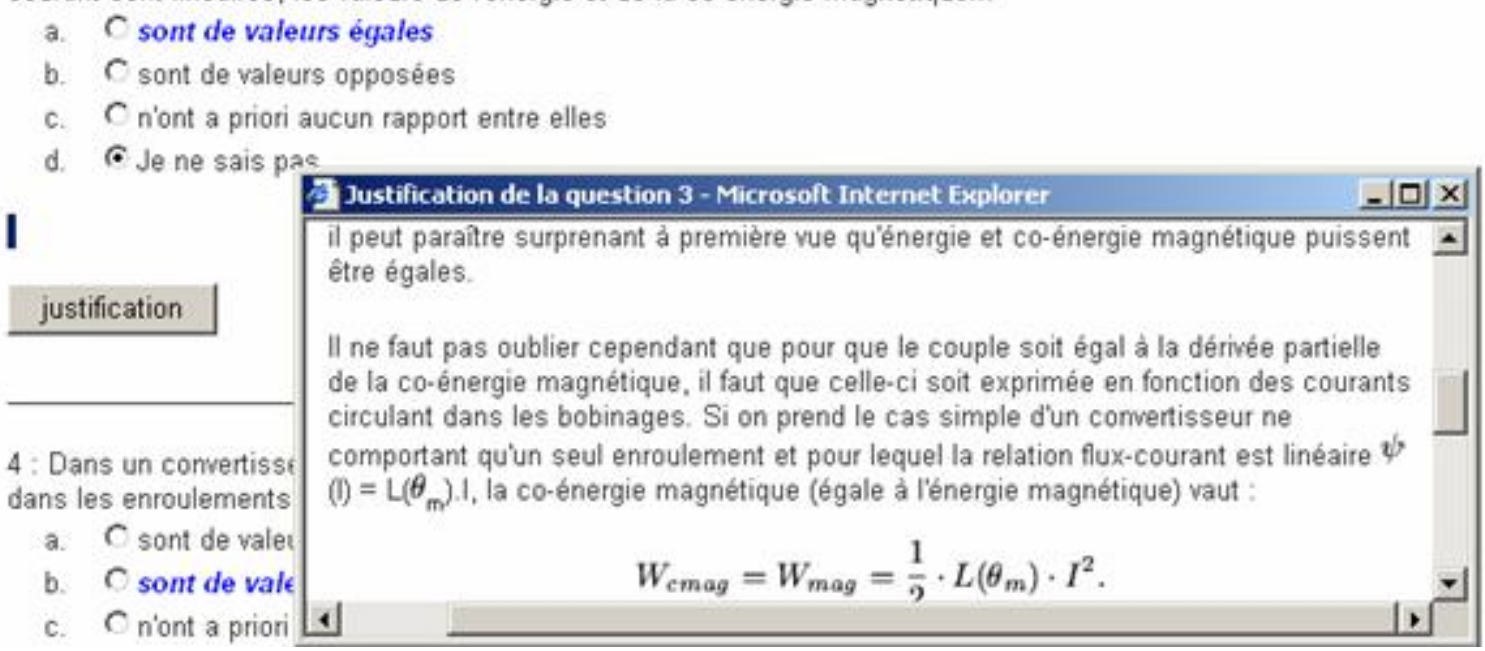

Figure 12. Page de correction d'un QCM.

Les sources java

Les applets sont des programmes écrits en langage java, encapsulés dans une page HTML. L'exécution de ce code java se fait via la "machine virtuelle" contenue dans les navigateurs compatibles (tel Microsoft Internet Explorer, Netscape ou Mozilla). Les étudiants peuvent donc, en salle didactique ou depuis leur ordinateur personnel, visualiser ces animations grâce à un simple navigateur internet, sans l'installation d'aucun programme spécifique.

Les sources java développées par les différentes équipes sont mises en commun. Une série de classes graphiques ont été programmées qui permettent l'affichage de graphes, de primitives graphiques telles que des vecteurs, phaseurs, amplitudes d'harmoniques, etc. mais également la gestion de fenêtres graphiques (rafraîchissement d'écrans, zoom, ...) permettent un développement plus rapide et plus aisé de nouvelles animations, même par des développeurs non spécialistes de la programmation java. 


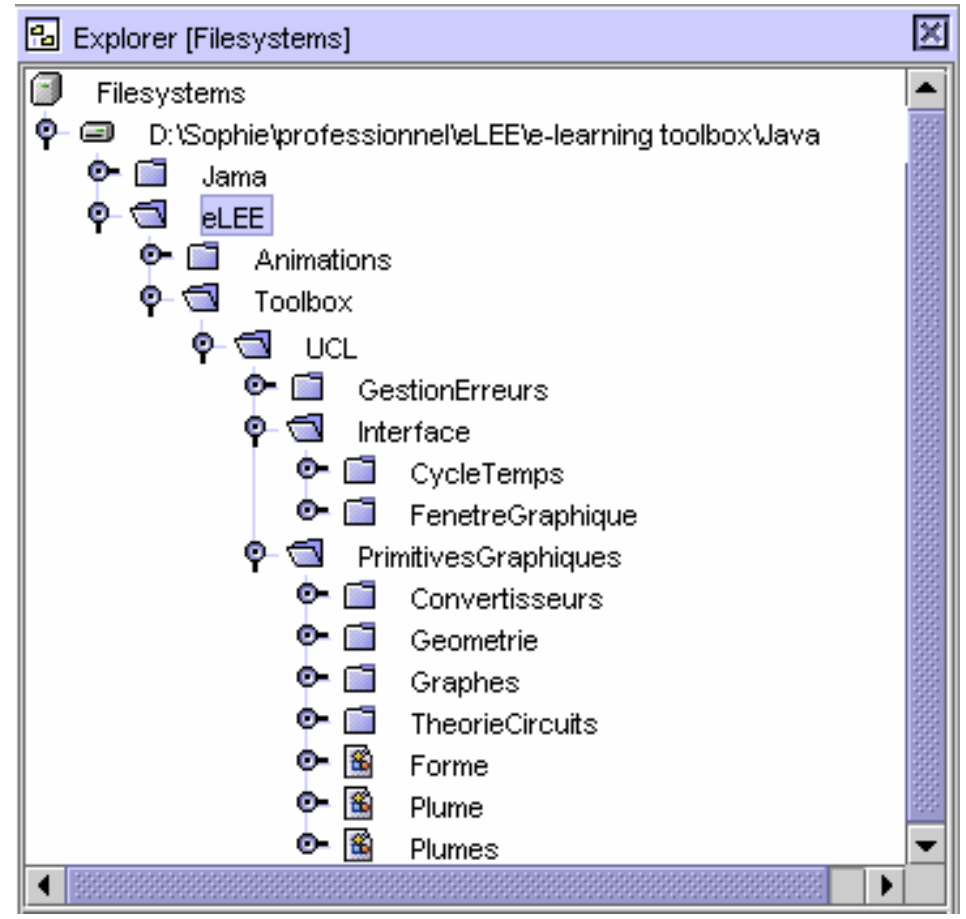

Figure 13a. Mise en commun des classes graphiques.

Ces classes sont, dans la mesure du possible, documentées : définition des variables, des méthodes (arguments et valeur retournée par la méthode).

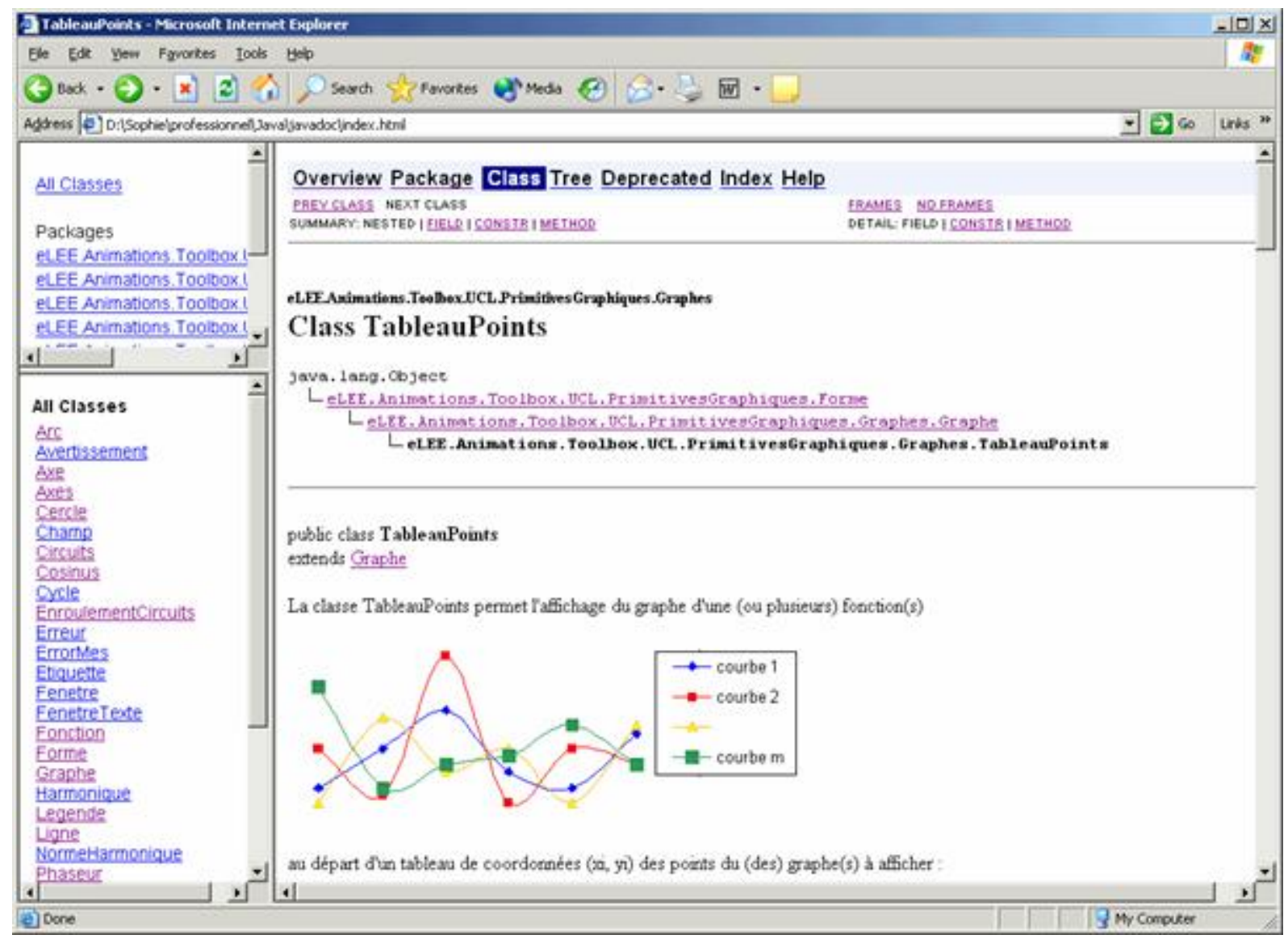

Figure 13b. Documentation des sources java. 
En ce qui concerne les simulations, un algorithme est proposé, qui modélise les circuits électriques par la méthode de la topologie pseudo-variable développée l'UCL [6],[7]. L'analyse de la topologie du système est effectuée selon une procédure proche de celle utilisée en mécanique multicorps et permet de gérer automatiquement les changements de topologie liés à la commutation des interrupteurs électronique de puissance. La traduction de cet algorithme en java est en cours de développement, une version beta (bibliothèque de composants réduite, certaines configurations non encore traitées dans l'algorithme) est disponible pour les partenaires du projet.

Les fiches de documentation

Des fiches de documentation sont régulièrement proposées sur le site. Elles permettent au développeur néophyte de s'initier rapidement aux méthodes et outils développés dans la "elearning toolbox".

Dans la mesure du possible, elles privilégient une approche "pas-à-pas" et tentent de répondre aux questions "classiques" que se posent les enseignants qui commencent à développer des ressources multimédia. 


\section{Q- LEE}

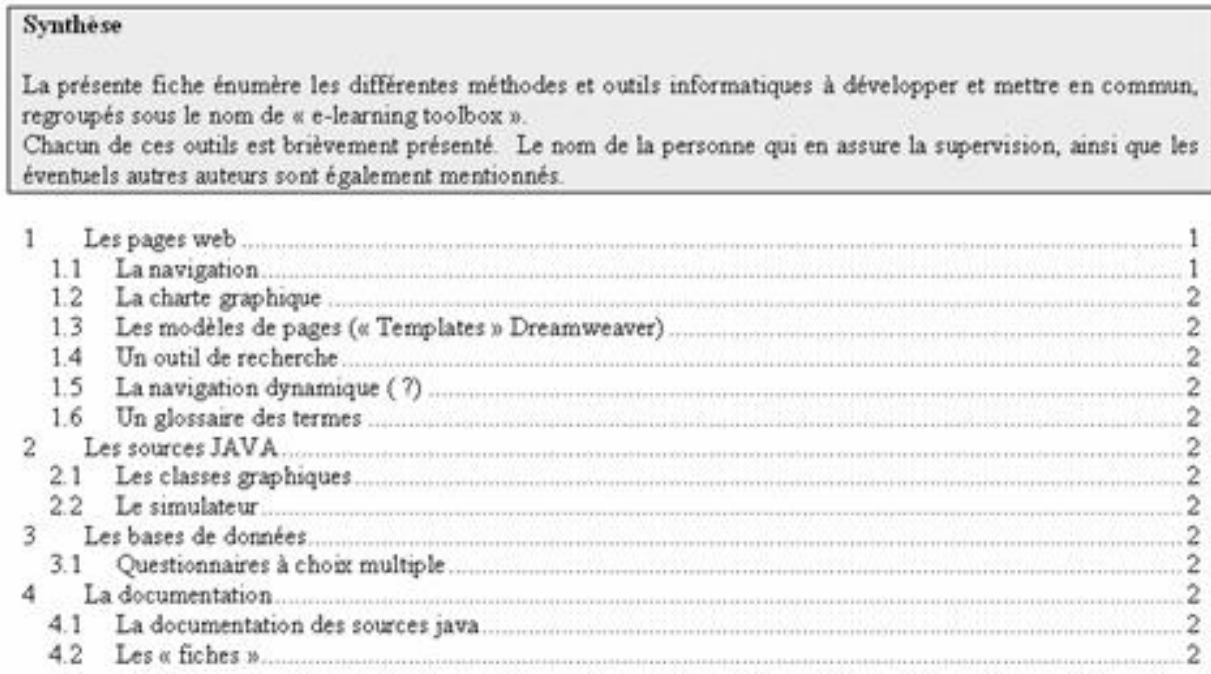

\section{Les pages web}

\subsection{La navigation}

Un modèle de navigation est proposé, organisé sur 4 niveaux. thématiques

chapitres, éventuellement subdivisés en

sections et enfin les

ressources (cours ou exposé, exercice / expérimentation virtuelle, questionnaires d'auto-tvaluation)

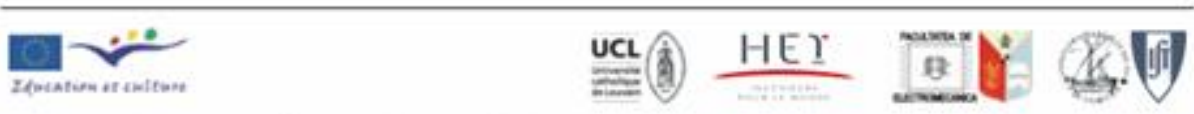

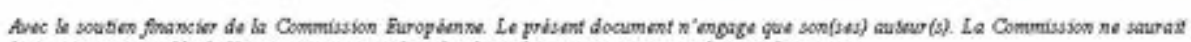

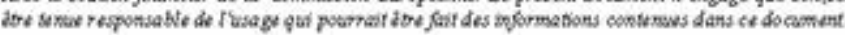

Figure 14. Les fiches de documentation.

L'hébergement du site

Enfin, l'hébergement des pages développées par les différentes équipes est géré par le coordinateur du projet (UCL), leur épargnant ainsi tous les problèmes liés à la gestion d'un site.

La structure finale du site ayant été planifiée au début du projet, les différentes versions du site, soit la traduction dans les différentes langues, ont été distinguées à la racine du 
répertoire. Les fichiers et répertoires ont été organisés suivant une structure hiérarchisée, identique dans les différentes versions. Chaque équipe de développeur a alors pris en main la gestion d'une (ou plusieurs) langue(s) du site.

Le programme Dreamweaver comprend une série de fonctions permettant de gérer un site et de transférer les fichiers depuis et vers un serveur distant. De cette façon, les différentes équipes peuvent récupérer les ressources développées par d'autres en vue d'en faire la traduction/adaptation. Ils peuvent également copier facilement et directement les fichiers des ressources qu'ils ont développées sur le serveur de l'UCL, au moyen du protocole FTP.

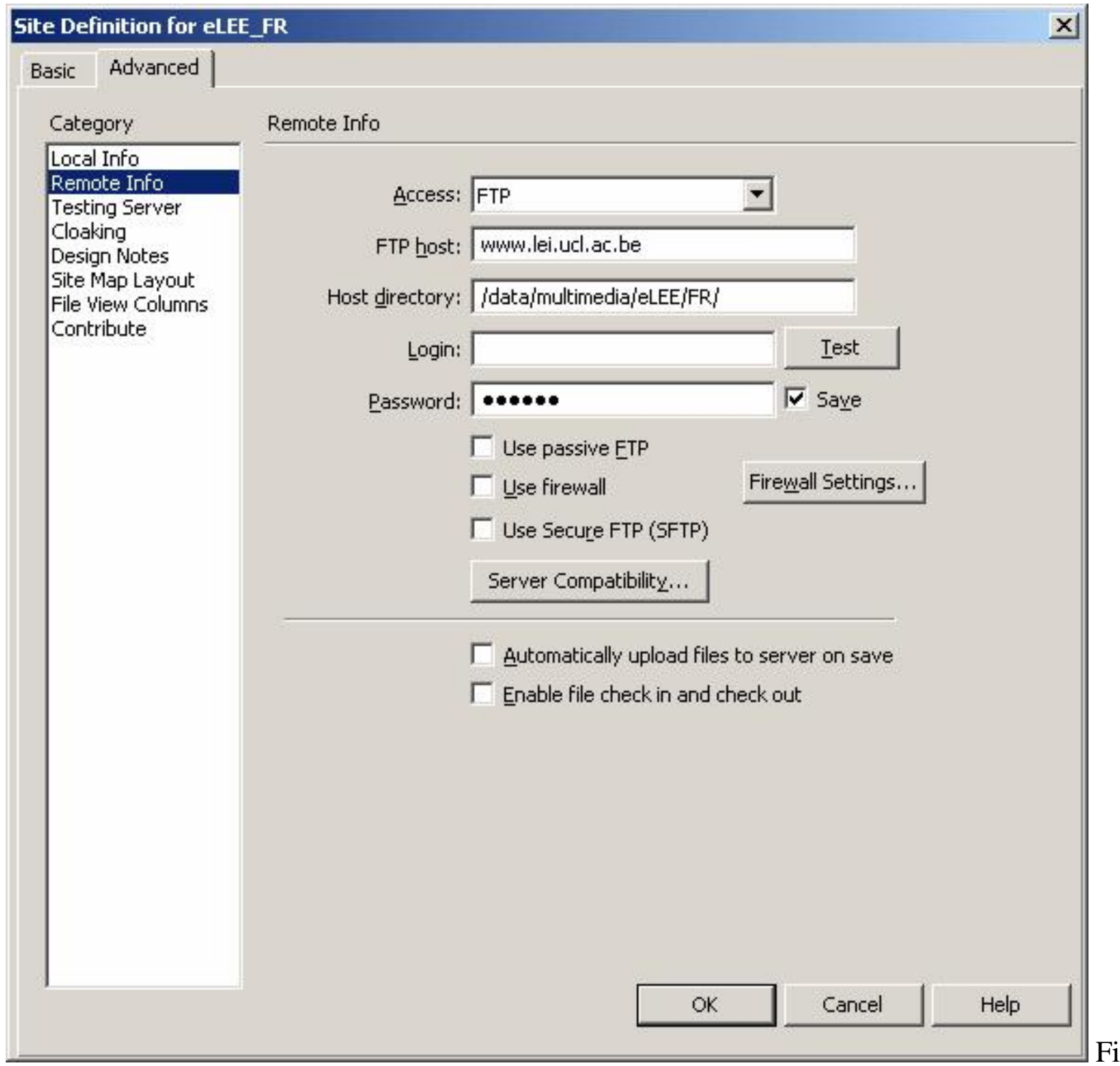

Figure 15. Gestion de site à distance dans Dreamweaver. 


\section{Création d'une association}

L'avantage de bénéficier de l'expérience acquise par d'autres sans l'obligation de devenir à la fois analyste-programmeur, webmaster et infographiste permet aux enseignants de se consacrer entièrement aux apports réels des TIC dans le processus d'apprentissage de leurs étudiants.

C'est d'ailleurs plus spécifiquement cet aspect qui a été analysé dans le processus d'évaluation également mené dans le cadre du projet "e-LEE". Si le lecteur est invité à lire d'autres articles [4], [5] qui présentent les principaux résultats de cette évaluation, les premières conclusions de l'enquête menée auprès des premiers concernés, à savoir les étudiants, montrent que l'usage des outils proposé dépendent des motivations personnelles des étudiants (comprendre la matière ou simplement réussir à court terme l'examen par exemple). Mais il dépend également fortement de l'attitude de l'enseignant, selon qu'il se contente de seulement renseigner le site ou l'intègre activement dans son dispositif pédagogique.

Le développement de telles ressources ne peut donc être envisagé que "sur mesure" ou du moins "adaptable". Elles peuvent difficilement être utilisées "telles quelles" par un autre enseignant, dans un autre établissement dont le niveau des étudiants, les objectifs et méthodes pédagogiques diffèrent. C'est pourquoi les partenaires du projet ont souhaité valoriser le travail qu'ils avaient effectué par le biais de la création d'une association sans but lucratif.

L'association e-LEE (e-learning tools for electrical engineering) a donc été créée à l'initiative des quatre partenaires du projet. Cette association poursuit trois objectifs principaux :

1. réaliser et diffuser gratuitement via un site internet (www.e-lee.net) des ressources multimédia pour l'enseignement du génie électrique ;

2. réaliser et diffuser sur le même site des études portant sur le profit que les étudiants peuvent tirer de telles ressources, fonction du contexte pédagogique, économique et social dans lequel ils évoluent ;

3. partager entre les membres de l'association les méthodes et outils informatiques permettant le développement de nouvelles ressources pédagogiques ou leur adaptation au contexte particulier d'enseignement.

L'association vise à coordonner les efforts de développement et sélectionner, par le biais de comités scientifiques ad hoc, les ressources développées par ses membres qu'elle juge utile de publier sur son site.

L'association est dotée d'une charte d'éthique et de déontologie. Cette charte précise notamment les règles relatives aux droits d'auteur des ressources développées. Tous les membres de l'association s'engagent à en respecter les dispositions. L'association cherche à élargir le partenariat actuel. Il s'agit :

- de recruter de nouveaux membres prêts à s'impliquer dans l'écriture de scenarii, la réalisation de nouvelles ressources ou la traduction / adaptation de ressources existantes ;

- d'impliquer des enseignants dans l'utilisation des ressources déjà développées ou à développer, recueillir l'avis de leurs étudiants et élargir ainsi notre base d'évaluation. Les conclusions que nous en tirerions n'en auraient que plus de pertinence. 


\section{Conclusion}

Partant du constat que le génie électrique est une discipline difficile à apprendre et à enseigner, quatre Universités et Grandes Ecoles développent un site d'e-learning avec le soutien de l'Union Européenne. Ce projet tente l'expérience d'un développement coopératif de ressources multimédia dans 4 thématiques : circuits électriques, électronique de puissance, actionnement asynchrone et énergies renouvelables. Les ressources seront déclinées en différentes langues. Différents types de ressources pédagogiques sont proposées : leçons en ligne, exercices résolus, laboratoires virtuels et questionnaires d'auto-évaluation... Les premiers résultats de l'évaluation de l'impact de ce nouvel outil auprès des étudiants ont encouragé les quatre partenaires du projet à créer une association sans but lucratif ayant pour objectif de poursuivre et élargir l'expérience, en y associant d'autres partenaires intéressés. 